\title{
Three-dimensional tumor model mimics stromal - breast cancer cells signaling
}

\author{
Stephanie Lemmo Ham ${ }^{1}$, Pradip Shahi Thakuri ${ }^{1}$, Madison Plaster ${ }^{1}$, Jun Li ${ }^{2}$, Kathryn \\ E. Luker ${ }^{3}$, Gary D. Luker ${ }^{3}$ and Hossein Tavana ${ }^{1}$ \\ ${ }^{1}$ Department of Biomedical Engineering, The University of Akron, Akron, $\mathrm{OH} 44325$, USA \\ ${ }^{2}$ Department of Mathematical Sciences, Kent State University, Kent, OH 44242, USA \\ ${ }^{3}$ Department of Radiology, Microbiology and Immunology, Biomedical Engineering, University of Michigan, Ann Arbor, MI \\ 48109, USA \\ Correspondence to: Hossein Tavana, email: tavana@uakron.edu \\ Gary D. Luker, email: gluker@umich.edu \\ Keywords: TNBC; tumor-stromal signaling; CXCL12; CXCR4; three-dimensional culture
}

Received: August 09, $2017 \quad$ Accepted: November 09, $2017 \quad$ Published: December 05, 2017

Copyright: Ham et al. This is an open-access article distributed under the terms of the Creative Commons Attribution License 3.0 (CC BY 3.0), which permits unrestricted use, distribution, and reproduction in any medium, provided the original author and source are credited.

\section{ABSTRACT}

Tumor stroma is a major contributor to the biological aggressiveness of cancer cells. Cancer cells induce activation of normal fibroblasts to carcinoma-associated fibroblasts (CAFs), which promote survival, proliferation, metastasis, and drug resistance of cancer cells. A better understanding of these interactions could lead to new, targeted therapies for cancers with limited treatment options, such as triple negative breast cancer (TNBC). To overcome limitations of standard monolayer cell cultures and xenograft models that lack tumor complexity and/or human stroma, we have developed a high throughput tumor spheroid technology utilizing a polymeric aqueous two-phase system to conveniently model interactions of CAFs and TNBC cells and quantify effects on signaling and drug resistance of cancer cells. We focused on signaling by chemokine CXCL12, a hallmark molecule secreted by CAFs, and receptor CXCR4, a driver of tumor progression and metastasis in TNBC. Using three-dimensional stromal-TNBC cells cultures, we demonstrate that CXCL12 - CXCR4 signaling significantly increases growth of TNBC cells and drug resistance through activation of mitogen-activated protein kinase (MAPK) and phosphoinositide 3-kinase (PI3K) pathways. Despite resistance to standard chemotherapy, upregulation of MAPK and PI3K signaling sensitizes TNBC cells in co-culture spheroids to specific inhibitors of these kinase pathways. Furthermore, disrupting CXCL12 - CXCR4 signaling diminishes drug resistance of TNBC cells in co-culture spheroid models. This work illustrates the capability to identify mechanisms of drug resistance and overcome them using our engineered model of tumor-stromal interactions.

\section{INTRODUCTION}

Signaling between cancer cells and tumor stroma drives all stages of cancer initiation and progression [1-4]. Histological examinations of human tumors show greater stromal content in more advanced and larger tumors, and high content of stromal cells correlates with greater risk of relapse and reduced survival [5-8]. Fibroblasts are the most abundant stromal cell type in epithelial tumors [9-12]. Cancer cells dynamically activate surrounding fibroblasts to produce growth factors, hormones, and cytokines that fuel tumor growth [13], and facilitate progression to metastasis [14]. These activated fibroblasts, termed carcinoma-associated fibroblasts (CAFs), regulate cancer cells through paracrine signaling and direct intercellular interactions. In a study of breast carcinoma, 
about 80 percent of fibroblasts exhibited an activated phenotype [15].

Production of the chemokine CXCL12 (also known as stromal cell-derived factor-1) is a hallmark feature of CAFs [16]. CXCL12 signals through CXCR4 and CXCR7 receptors upregulated on cancer cells [17], and activates multiple molecular pathways such as the mitogen-activated protein kinase (MAPK), inositol 1,4,5-triphosphate (IP3), and phosphoinositide 3-kinase (PI3K) [18]. Signaling through these pathways promotes survival, proliferation, metastasis, and drug resistance of cancer cells [18-21]. The CXCL12 - CXCR4/CXCR7 signaling axis is highly active in breast cancers [22, 23]. Analysis of human breast tumors showed that among the different molecular subtypes, triple negative breast cancer (TNBC) cells have the most elevated expression of CXCR4 [24]. Considering the lack of targeted therapies for TNBC [25], disrupting this chemokine signaling may offer a potential new therapeutic approach. In addition, investigating pathways downstream of this chemokine signaling axis in TNBC cells potentially will unveil new molecular targets for therapy.

Studies of interactions among stroma and cancer cells typically use monolayer cell cultures or xenograft models. Monolayer cultures lack the complexity of tumors including a compact three-dimensional morphology, close intercellular contacts, exposure of cells to gradients of soluble factors and oxygen, and nonuniform, spatially-dependent cell proliferation [26-28]. Thus, cells in monolayer cultures often fail to reproduce key characteristics of tumors. Xenografts present a physiological system for cancer research. However, they are limited in terms of absence of human tumor stroma, failure of some murine cytokines to activate human receptors, disparities in dynamics of tumor growth and progression compared to human tumors, and need for complex tools and biological assays to study stromal effects on cancer cells [29-31]. Since the introduction of tissue microarrays and next generation sequencing [32], these technologies have been used for high throughput assessment of biomarkers in thousands of tumor samples from biopsies using standard assays such as immunohistochemistry and fluorescent in-situ hybridization [33]. These technologies are an invaluable tool in clinical oncology to develop diagnostic tests and identify disease biomarkers. Nevertheless, tissue microarrays and similar biopsy-based assays for endpoint analysis of fixed samples do not capture dynamic tumorstromal interactions in tumor microenvironments. Tumors constantly transform both spatially and temporally. Interactions between stromal and cancer cells play a major role in conferring functional changes to cancer cells such as gain of cancer stem cell and mesenchymal characteristics, altered metabolism, drug resistance, migration and invasion, and survival [34]. Mechanistic understanding of these complex events in tumors requires approaches that reproduce dynamic signaling of cancer and stromal cells as it occurs in native tissues.

Three-dimensional (3D) cultures of cells as spheroids provide relevant in vitro tumor models to recapitulate architecture and complex intercellular network of tumors and emulate stromal-cancer cells interactions [26, 35-37]. We recently developed a robotic, high throughput spheroid microprinting technology to mass produce homogenouslysized spheroids that exhibit key biology properties of solid tumors [38-41]. Here, we utilized this technology and formed an array of co-culture spheroids of TNBC and stromal cells to examine CXCL12 signaling through CXCR4 and CXCR7 receptors on TNBC cells. Using different cellular assays and molecular analyses, we demonstrated that CXCL12 - CXCR4 signaling significantly increases spheroid proliferation and TNBC cell growth. This signaling conferred resistance to standard chemotherapy drug treatment through activation of MAPK and PI3K pathways. We found that CXCL12 CXCR4 signaling induces sensitivity of the cancer cells to specific molecular inhibitors of MAPK and PI3K pathways, preventing proliferation of TNBC cells. This work establishes the feasibility of studying tumor-stromal interactions using our engineered solid tumor models and offers a convenient preclinical tool to identify new treatment approaches.

\section{RESULTS AND DISCUSSION}

\section{Aqueous two-phase system (ATPS) microprinting of TNBC-stromal cells co-culture spheroids}

The ATPS technology facilitates partitioning of cancer and stromal cells to the DEX phase nanodrop to spontaneously form a mono-culture or a co-culture spheroid within 24-48 hours of incubation (Figure 1A-1B) $[42,43]$. Importantly, nutrients and waste products of cells freely diffuse between the DEX phase nanodrop and the immersion PEG phase [38]. Adapting the technology to robotics enabled formation of spheroids in standard 384-microwell plates [44]. For co-culture spheroids, we selected a ratio of $1: 2 \mathrm{TNBC}$ to stromal cells and a total cell density of $1.5 \times 10^{4}$ cells $/ 0.3 \mu 1$ of DEX phase drop. This ratio was to mimic more advanced and larger human breast tumors that have greater stromal content than cancer cells $[5,6,45,46]$. Using larger ratios of $1: 3$ and 1:4 (breast cancer cells to fibroblasts) while keeping the initial breast cancer cell density constant at $5 \times 10^{3}$ cells per DEX phase drop did not alter growth of TNBC cells (Supplementary Figure 1), consistent with other studies $[47,48]$. This microprinting approach gave consistentlysized mono-culture spheroids of $\mathrm{CXCR} 4^{+} \mathrm{TNBC}$ cells $\left(5 \times 10^{3}\right.$ cells), mono-culture spheroids of fibroblast cells, HMF and CAFs, $\left(1 \times 10^{4}\right.$ cells $)$, and co-culture spheroids of CXCR $4^{+}$TNBC cells with HMF cells or CAFs $\left(1.5 \times 10^{4}\right.$ cells with a 1:2 TNBC to stromal cells ratio) (Figure 1C). The spheroid size consistency was measured from two 
separate experiments to ensure that spheroids of each model had a similar initial metabolic activity baseline. Importantly, the $1.5 \times 10^{4}$ cell density co-culture spheroids containing HMF cells or CAFs were not statistically different in size $(p>0.05)$, eliminating potential effects of size differences of the spheroids on the studies reported below. We conveniently maintained spheroids in the same 384-microwell plate used for spheroid formation by robotic exchange of culture medium.

\section{Effect of TNBC-stromal cells signaling on cellular metabolic activity of spheroids}

To investigate the effect of CXCL12 signaling through CXCR4 and/or CXCR7 receptors on the proliferation of cells in 3D cultures, we generated eight different co-culture spheroids that contained at least one element of this chemokine-receptor(s) signaling or completely lacked them: (i) TNBC:HMF, (ii) TNBC:CAFs, (iii) $\mathrm{CXCR} 4^{+} \mathrm{TNBC}: \mathrm{HMF}$, (iv) $\mathrm{CXCR}^{+} \mathrm{TNBC}: \mathrm{CAFs}$, (v) $\mathrm{CXCR}^{+} \mathrm{TNBC}: \mathrm{HMF}$, (vi) $\mathrm{CXCR}^{+} \mathrm{TNBC}$ :CAFs, (vii) $\mathrm{CXCR}^{+} / \mathrm{CXCR}^{+} \mathrm{TNBC}: \mathrm{HMF}$, and (viii) $\mathrm{CXCR}^{+} /$ CXCR7 ${ }^{+}$TNBC:CAFs. The co-culture spheroids of TNBC:HMF that lacked this signaling served as the global negative control. Spheroids were evaluated for their cellular proliferation over a six-day period following formation and in medium containing only $1 \% \mathrm{FBS}$ at the starting point and no renewal (Figure 2A). The TNBC:CAFs model displayed either a lower or fairly similar activity compared to the TNBC:HMF model, indicating that CXCL12 production by CAFs does not confer growth advantage to TNBC cells lacking the cognate receptors of the chemokine. On the other hand, the CXCR4 $4^{+}$TNBC:CAFs model showed overall the highest and continuously increasing activity throughout the culture compared to all the other co-culture spheroids. The CXCR4 $4^{+} \mathrm{TNBC}$ :CAFs model also consistently showed $11-28 \%$ greater activity than its CXCR4+TNBC:HMF counterpart lacking the CXCL12 chemokine as determined by a linear regression analysis. There was no significant difference between cell proliferation in CXCR $7^{+} \mathrm{TNBC}: \mathrm{HMF}$ and CXCR7 $7^{+}$TNBC:CAFs co-culture spheroids. Interestingly, these models even had lower activity level than the TNBC:HMF and TNBC:CAFs spheroids on each day of measurement. When the effect of expression of both CXCR4 and CXCR7 receptors on cell proliferation was evaluated, the $\mathrm{CXCR}^{+} / \mathrm{CXCR} 7^{+} \mathrm{TNBC}$ :CAFs model had slightly increased activity, up to $4.5 \%$, than the $\mathrm{CXCR}^{+} /$ CXCR $7^{+}$TNBC:HMF spheroids on certain days. Our statistical analysis of the multivariate, temporally-dependent data resulting from all eight co-culture spheroid models showed that cell proliferation of the $\mathrm{CXCR} 4^{+} \mathrm{TNBC}$ :CAFs model was significantly different from the seven other models across the six-day culture period (Figure 2B).

Consistent with reports in mouse models [49-51], our finding suggests that the CXCR4 - CXLC12 axis is a key mediator of cell proliferation in breast cancer. The effect of CXCR4 - CXLC12 signaling on promoting primary and metastatic breast tumor growth has been demonstrated in animal models and clinical studies $[52,53]$. Several studies have reported that CXCR7 is a scavenger receptor to prevent saturation of CXCR4 receptors and also to create CXCL12 gradients to facilitate migration of CXCR4 ${ }^{+}$cancer cells [17, 54-57]. This provides a plausible explanation for the similar activity levels of the CXCR7 $7^{+} \mathrm{TNBC}: \mathrm{HMF}$ and CXCR7 ${ }^{+}$TNBC:CAFs co-culture models. We note that the role of the CXCR7 receptor in the presence of CXCL12

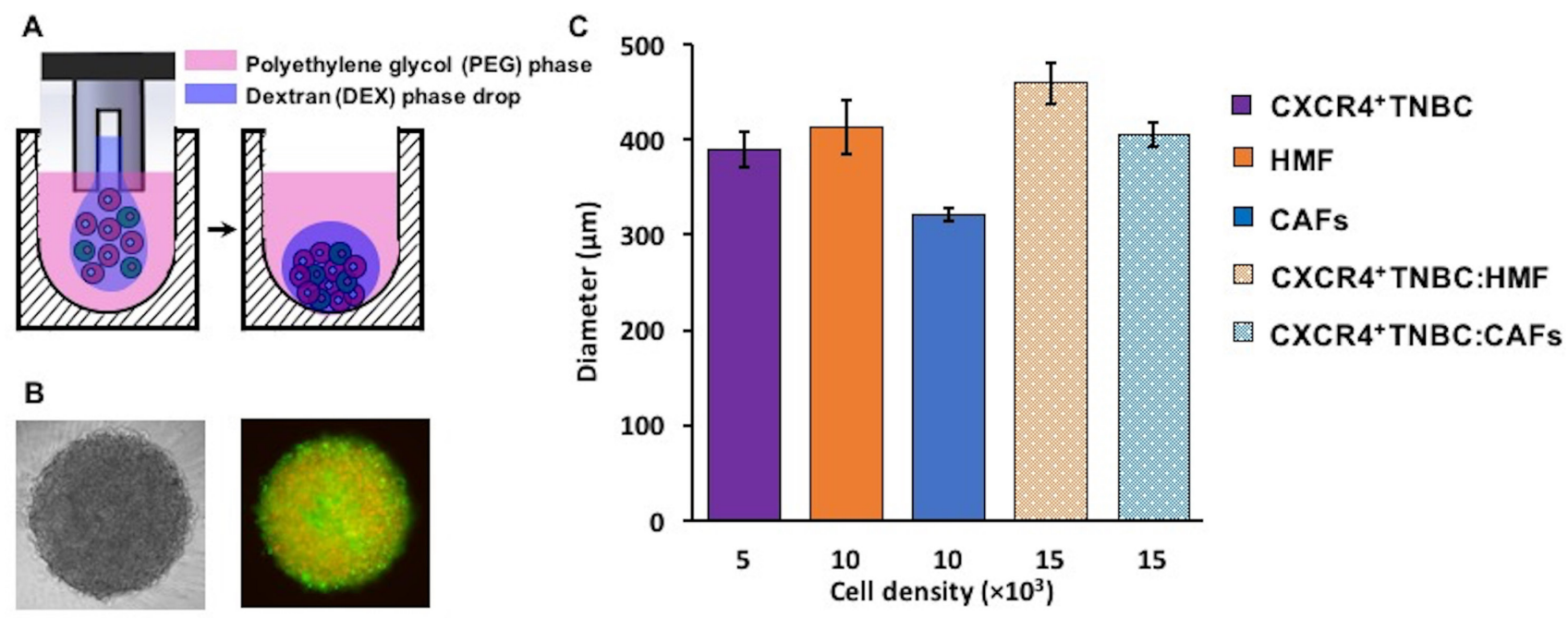

Figure 1: (A-B) Cancer cells remain confined in the $0.3 \mu 1$ DEX phase drop (purple) suspended in the immiscible immersion PEG phase (pink) and autonomously aggregate to form a co-culture spheroid of triple negative breast cancer cells (green) and human mammary fibroblasts (red) in $48 \mathrm{hrs}$. Colors in panel (A) are for presentation purpose only. (C) Resulting spheroids of different co-culture models are consistently sized with low standard errors. 
signaling remains disputed [58-60]. For example, studies show that CXCR7 expression lead to growth of primary breast tumors in rat models through promoting angiogenesis, which is consistent with data showing upregulation of this receptor on tumor vasculature [61-63]. However, results from these studies cannot be correlated with those from our 3D cultures that represent models of avascular tumors. Altogether, these data demonstrate that our 3D cultures recapitulate growth properties of breast tumors. Based on analysis of the data, we selected the CXCR4 $4^{+}$TNBC:CAFs model and its respective negative control, CXCR4 ${ }^{+} \mathrm{TNBC}: \mathrm{HMF}$, for mechanistic studies of CXCR4 - CXLC12 signaling in TNBC.

\section{Inhibition and stimulation of CXCL12 - CXCR4 signaling}

We conducted two sets of experiments to evaluate if the higher proliferative activity of the CXCR4 ${ }^{+}$TNBC:CAFs model was indeed due to
CXCR4 - CXLC12 signaling. The experiments included an inhibition test and a stimulation test. First, we treated spheroids with AMD3100, an antagonist of CXCL12 - CXCR4 that blocks the CXCR4 receptor $[64,65]$. Consistently throughout the six-day culture, this treatment reduced proliferation of the CXCR4 ${ }^{+}$TNBC:CAFs model to the level of the $\mathrm{CXCR} 4^{+} \mathrm{TNBC}: \mathrm{HMF}$ model that lacks CXCL12 (Figure 3A). Next, we treated the CXCR4 $4^{+} \mathrm{TNBC}: \mathrm{HMF}$ model with CXCL12-containing conditioned medium of CAFs. This stimulation elevated the proliferation of the CXCR4 ${ }^{+}$TNBC:HMF model to the level of the CXCR4 ${ }^{+}$TNBC:CAFs model (Figure 3B). In both cases, our analysis showed minimal statistically significant differences between the treated and non-treated co-culture models. Collectively, these results established that greater proliferation of the CXCR4 ${ }^{+}$TNBC:CAFs spheroid model than the CXCR4 ${ }^{+}$TNBC:HMF spheroid model was due to CXCR4 - CXCL12 signaling.

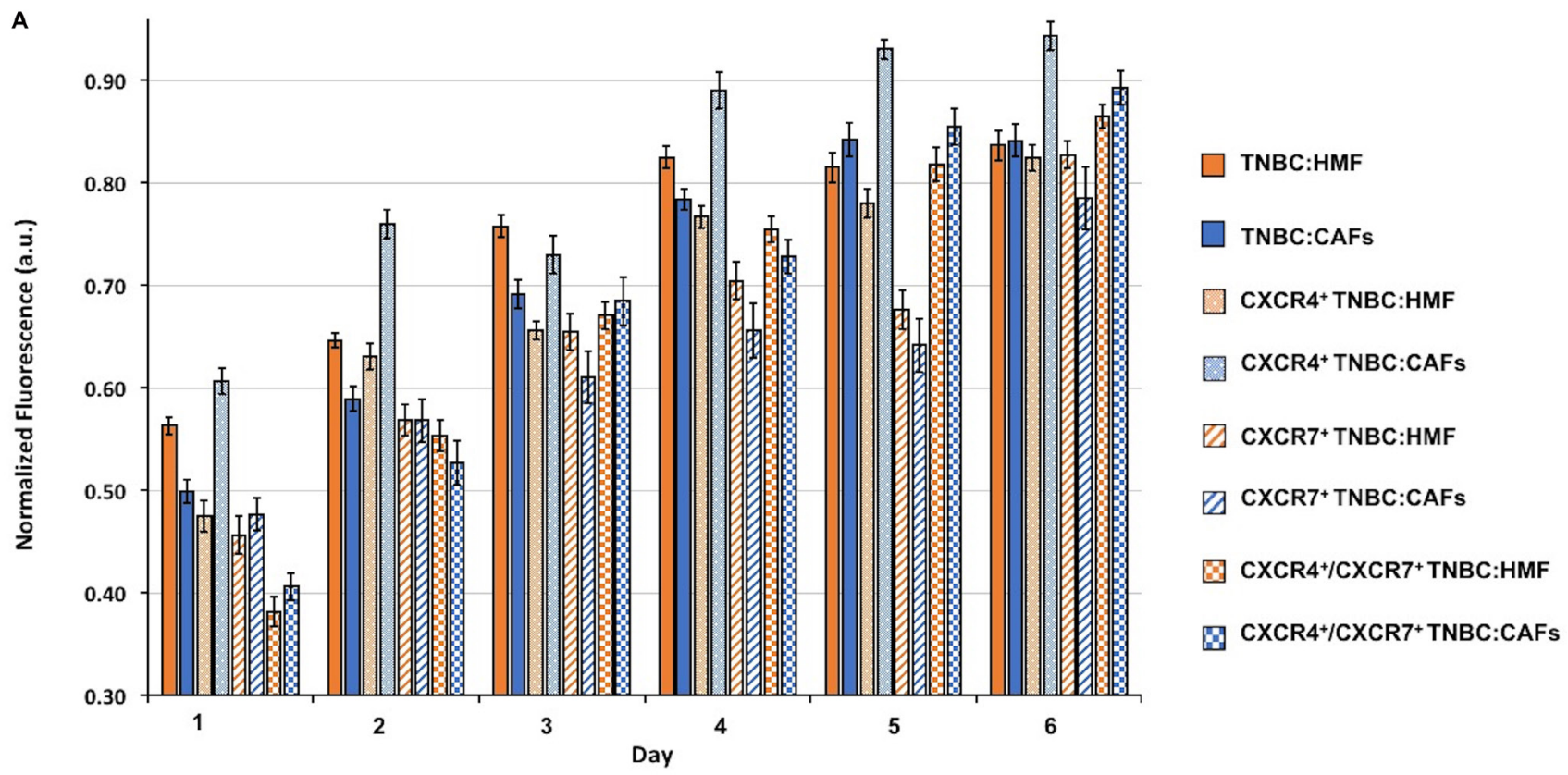

B

\begin{tabular}{|c|c|}
\hline $\begin{array}{l}\text { Co-cultures compared } \\
\text { against CXCR4+TNBC:CAF }\end{array}$ & $p$-value \\
\hline TNBC:HMF & $7.37 \times 10^{-6}$ \\
\hline TNBC:CAFS & $1.49 \times 10^{-4}$ \\
\hline CXCR4 $4^{+}$TNBC:HMF & $5.02 \times 10^{-8}$ \\
\hline CXCR7' TNBC:HMF & $7.56 \times 10^{-10}$ \\
\hline CXCR7 $7^{+}$TNBC:CAFs & $1.12 \times 10^{-11}$ \\
\hline $\mathrm{CXCR}^{+} / \mathrm{CXCR} 7^{+} \mathrm{TNBC}: \mathrm{HMF}$ & $1.10 \times 10^{-8}$ \\
\hline CXCR4 $4^{+} / \mathrm{CXCR} 7^{+}$TNBC:CAFs & $5.02 \times 10^{-8}$ \\
\hline
\end{tabular}

Figure 2: (A) Growth of co-culture spheroids based on metabolic activity measurements is shown over a six-day period. Spheroids consist of $1.5 \times 10^{4}$ cells at a ratio of 1:2 TNBC:fibroblasts at the starting point of experiments. (B) $p$-values from a statistical test show that the CXCR4 ${ }^{+}$TNBC:CAFs co-culture model is consistently and significantly more proliferative than all the other models. 


\section{Evaluation of cancer cell growth in spheroids due to CXCL12 - CXCR4 signaling}

The above study based on metabolic activity of cells provided a measure of stromal-cancer cells signaling effect on the overall growth of spheroids. Considering that activated stromal cells (CAFs) in breast tumors also proliferate, we investigated to what extent greater overall growth results from proliferation of $\mathrm{CXCR} 4^{+} \mathrm{TNBC}$ cells in CXCR4 ${ }^{+}$TNBC:CAFs spheroids compared with other spheroid models shown in Figure 2A. Measurements of the endogenous eGFP signal of the cancer cells showed that cancer cells in the CXCR4 $4^{+} \mathrm{TNBC}: \mathrm{CAFs}$ model display a statistically significant $(p<0.05)$ growth increase throughout the measurement window (Figure 4A). Linear regression analysis showed that $\mathrm{CXCR} 4^{+} \mathrm{TNBC}$ cells in this model had an $11.4 \%$ larger growth slope over time than their counterparts in the CXCR4 $4^{+} \mathrm{TNBC}: \mathrm{HMF}$ model. This indicates that CAFs confer increasingly greater proliferative activity to the breast cancer cells. To validate this result, we evaluated growth of cancer cells in co-culture spheroids of TNBC:HMF and TNBC:CAFs that lacked both elements of the signaling axis or CXCR4 receptor expression, respectively. Despite some random differences in growth of cancer cells between the two models on several days, the slope of linear regression was smaller by $2 \%$ in the co-culture containing CAFs (Figure 4B). Thus, these results established the role of CXCR4 CXCL12 signaling on enhanced growth of breast cancer cells $[66,67]$.

TNBC cells in all four co-culture spheroid models displayed a larger growth than their respective monoculture spheroids generated with the same density of $5 \times 10^{3}$ breast cancer cells. The slope of linear regression of growth curves was 5.0 folds and 1.8 folds larger for the CXCR4 ${ }^{+}$TNBC:CAFs and TNBC:CAFs co-culture models relative to their mono-culture breast cancer cell spheroids, respectively. Similarly, the regression line slope was 4.4 folds and 1.8 folds larger for the CXCR4 $4^{+} \mathrm{TNBC}$ :HMF and TNBC:HMF co-culture models relative to their monoculture breast cancer cell spheroids, respectively. This implies a major role for heterotypic cellular signaling in tumors and suggests that breast cancer cells induce the normal fibroblasts to produce soluble factors to promote growth of malignant cells. Studies show that cancer cells produce growth factors such as transforming growth factor beta (TGF- $\beta$ ), platelet-derived growth factor (PDGF), and fibroblast growth factor 2 (FGF2) that activate the adjacent stromal cells, which in turn secrete signaling molecules such as hepatocyte growth factor (HGF) to promote proliferation of cancer cells [10, 16, 68-71]. The larger growth rate of cancer cells in the CXCR4 ${ }^{+} \mathrm{TNBC}: \mathrm{HMF}$ co-culture spheroids compared to the respective CXCR4 ${ }^{+}$TNBC mono-culture spheroids may be mediated by soluble factors secreted by CXCR4-expressing cancer cells to transform HMF cells to CAFs [72].

Morphologically, mono-culture spheroids of CXCR4 ${ }^{+}$TNBC and TNBC became more compact by day six of culture. Co-culturing breast cancer cells and fibroblasts significantly enhanced compactness of spheroids earlier, indicating that fibroblasts facilitate spheroid formation of breast cancer cells (Figure 4C-4D) [73]. The co-culture models containing CXCR4 $4^{+} \mathrm{TNBC}$ cells exhibited a compact morphology for several days with minimal increases of volume (9-15\%) from days 1 to 3 . But over time, the co-culture spheroids of CXCR $4^{+}$TNBC and HMF cells showed separation of fibroblast and cancer cells with an $82 \%$ volume increase from days 3 to 6 . This is likely due to the expression of incompatible junctional proteins in breast cancer and fibroblast cells [74]. For example, it was shown that intercellular adhesion was dependent on cadherins expression of each cell type,

A AMD3100 treatment

B CXCL12 treatment

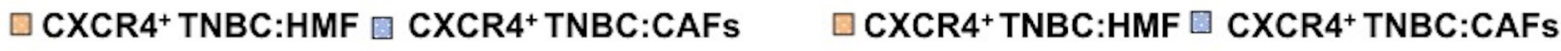
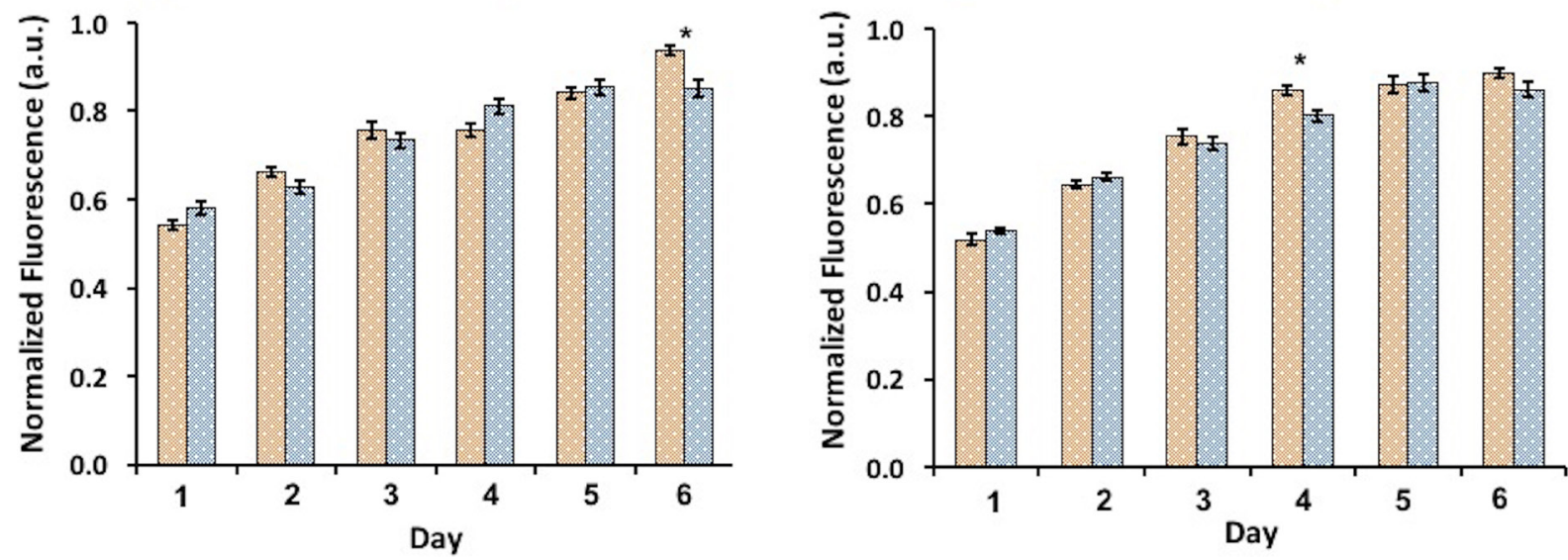

Figure 3: (A) Treatment of CXCR4 ${ }^{+}$TNBC:CAFs co-culture spheroids with AMD3100 (CXCR4 antagonist) normalizes proliferation to level of the CXCR4 ${ }^{+}$TNBC:HMF co-culture. (B) Treating CXCR4 ${ }^{+}$TNBC:HMF co-culture spheroids with CXCL12-containing conditioned medium from CAFs stimulates proliferation to that of CXCR4 ${ }^{+} \mathrm{TNBC}$ :CAF co-culture spheroids. 
and only cells that expressed the same type of cadherins intermixed [75]. Notably, the separation behavior was observed considerably more in the TNBC:HMF co-culture model and was further demonstrated through confocal imaging in Figure 4E-4F. Expression of CXCR4 on TNBC cells largely prevented separation from HMF cells in the CXCR4 ${ }^{+}$TNBC:HMF model. Additionally, inclusion of CAFs, regardless of CXCR4 expression on TNBC cells, blocked the segregation effect and helped the spheroids retain a compact morphology, mimicking the unique characteristic of CAFs to infiltrate tumor masses [76]. All spheroids displayed shedding of TNBC cells from their peripheries on later days of culture, suggesting that active proliferation of border cells is somewhat compensated by cell shedding [77]. We note that a thorough understanding of the underlying mechanisms of these morphological differences in co-culture spheroids requires a more extensive analysis.
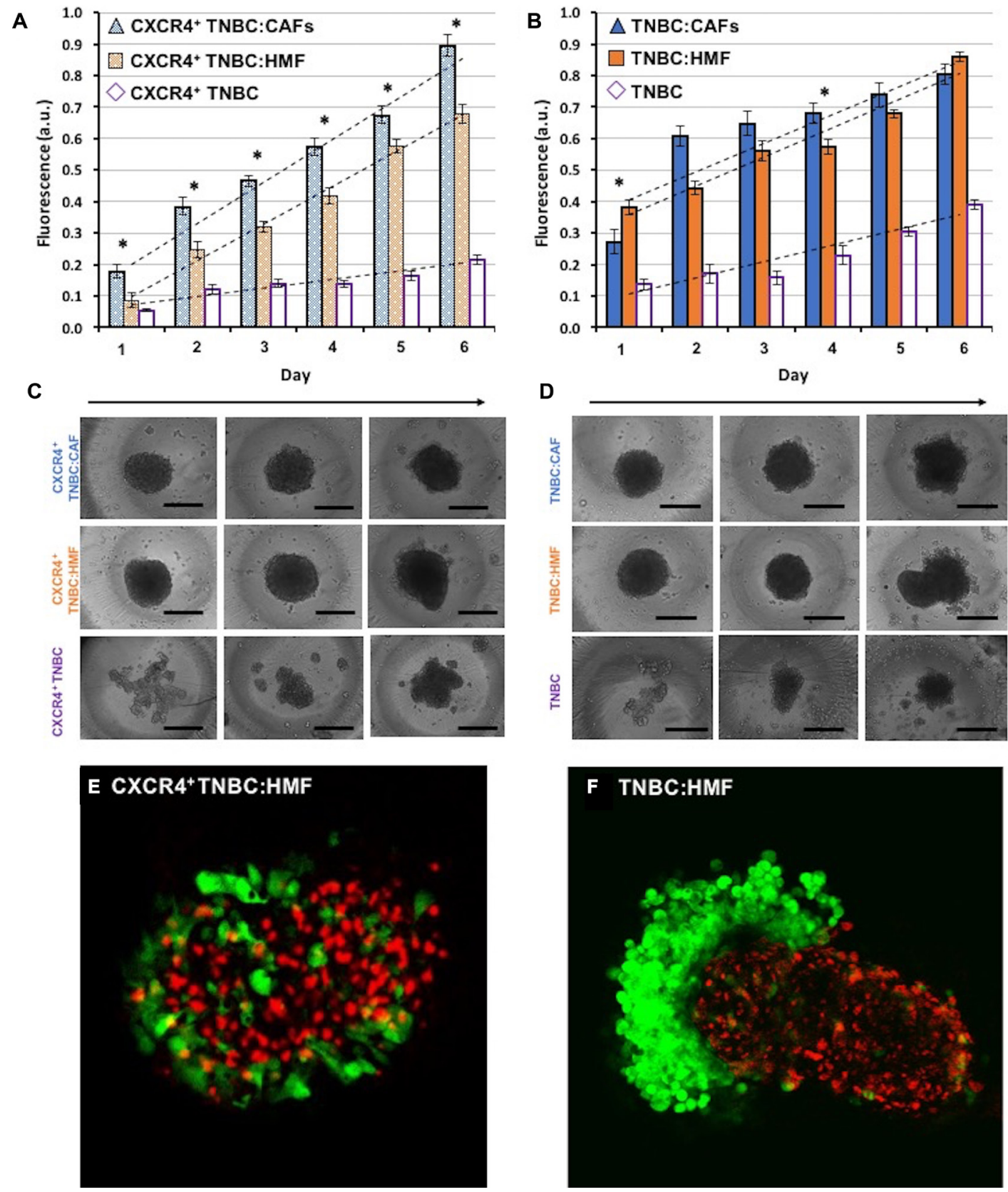

Figure 4: Proliferation of TNBC cells in co-culture spheroids measured using their endogenous eGFP signal. (A) TNBC cells in the CXCR4 ${ }^{+}$TNBC:CAFs model display consistently greater proliferation compared to spheroids of CXCR4 ${ }^{+} \mathrm{TNBC}: \mathrm{HMF}$ coculture model and CXCR4 ${ }^{+}$TNBC single culture with a larger slope of $11.4 \%$ and $77.1 \%$, respectively. (B) TNBC cells lacking CXCR4 expression in co-culture with HMF and CAFs show a similar proliferation. (C-D) Morphology of resulting co-culture spheroids and mono-culture spheroids of breast cancer cells on days 1, 3, and 6 (left to right). Error bars represent standard error of mean. (E-F) Confocal images of the co-culture spheroids containing HMF cells on day 4. Scale bar is $200 \mu \mathrm{m} .{ }^{*} p<0.05$. 


\section{Molecular analysis of CXCL12 - CXCR4 signaling on cancer cell proliferation in spheroids}

We evaluated proliferation of both breast cancer and stromal cells in the CXCR4 ${ }^{+}$TNBC:CAFs and CXCR4 ${ }^{+}$TNBC:HMF co-culture spheroids using IHC analysis of cryosections of spheroids and quantifying $\mathrm{Ki}-67^{+}$proliferative cells. Breast cancer and stromal cells showed statistically significant higher proliferation $(p<0.05)$ in the CXCR $4^{+}$TNBC:CAFs model (Figure 5). When co-cultured with CAFs, the CXCR4 ${ }^{+}$TNBC cells showed 1.4 times greater Ki-67 staining, in agreement with results based on eGFP signal measurements (Figure 4). Additionally, CAFs in the co-culture model had a remarkably two-fold higher Ki-67 staining compared to HMF cells. This is consistent with increased proliferation of CAFs in tumor tissues [10,78], and the overall greater stromal content in more aggressive and larger tumors
$[6,79,80]$. We note that the CAFs secrete mCherrytagged CXCL12 into the medium, whereas the HMF cells contain mCherry signal in their nuclei. This is the reason for visual differences between the red fluorescence images of CAFs and HMF cells in Figure 5. To avoid relying on the mCherry signal for quantification of cell proliferation, we used the Ki- $67^{+}$and eGFP signal of breast cancer cells to quantify proliferative TNBC cells, and then subtracted this value from the total $\mathrm{Ki}-67^{+}$fluorescence to quantify proliferation of stromal cells (CAFs or HMF) in their respective co-cultures.

Next, we investigated molecular mechanisms for increased proliferation of stromal and breast cancer cells in the CXCR4 ${ }^{+}$TNBC:CAFs spheroid model. The CXCL12 - CXCR4 axis may activate multiple pathways in cancer cells [17, 81-83]. We focused on ERK and AKT, two prominent drivers of proliferation in breast cancer and other malignancies [84]. Western blot analysis relative to the total protein and $\beta$-actin expression of each
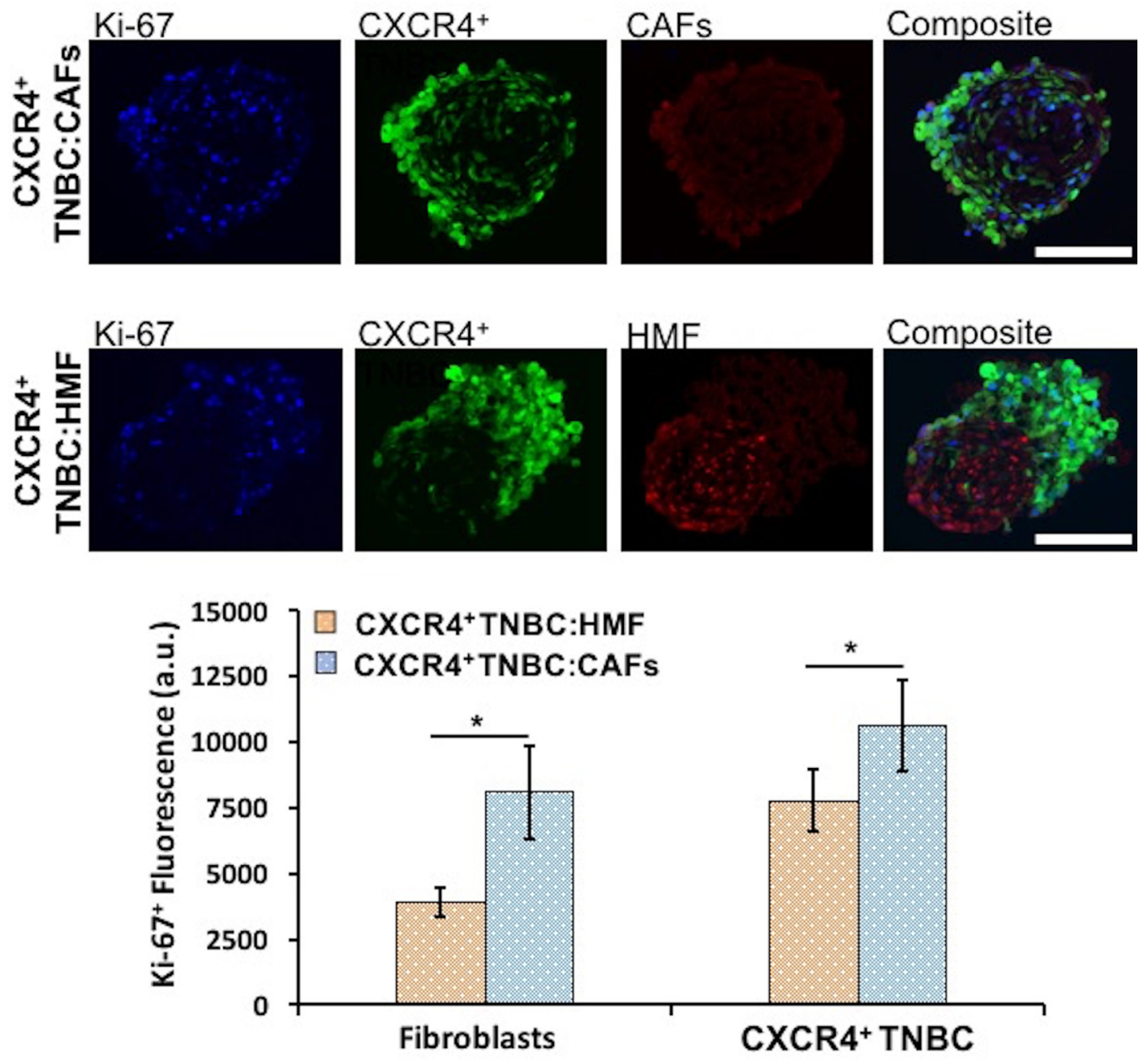

Figure 5: Proliferation of $\mathrm{CXCR} 4{ }^{+} \mathrm{TNBC}$ cells and fibroblasts (HMF and $\mathrm{CAFs}$ ) in co-culture spheroids characterized through measurements of $\mathrm{Ki}-67^{+}$proliferative cells using immunostained images of cryosections of 4-day old spheroids. Both cancer cells and fibroblasts show significantly greater proliferation in the CXCR4 ${ }^{+}$TNBC:CAFs co-culture model (blue bars). Scale bar is $200 \mu \mathrm{m} .{ }^{*} p<0.05$. 
co-culture system showed that cells in CXCR4 ${ }^{+}$TNBC:CAFs spheroids had moderately higher levels of $p$-ERK (Figure 6A) and substantially higher levels of $p$-AKT (Figure $6 \mathrm{~B}$ ) compared to CXCR4 ${ }^{+} \mathrm{TNBC}: \mathrm{HMF}$ spheroids. The displayed immunoblots were quantified (normalized to their respective total and $\beta$-actin protein expressions), showing an $81 \%$ increase in the $p$-AKT $/ t$ AKT levels and a $16 \%$ increase in $p$-ERK $/ t$-ERK levels in the CXCR4 $4^{+} \mathrm{TNBC}$ :CAFs spheroids. Although $p$-ERK increase is modest, small changes in activation of MAPK components produce much larger effects on overall signaling and biologic outputs [85]. These results suggest that the CXCL12 - CXCR4 signaling upregulates activation of the PI3K and MAPK pathways to support TNBC cells metabolic and growth activities.

To further substantiate these results, we conducted IHC analysis of cryosections of co-culture spheroids for ERK and AKT phosphorylation (Figure 7A-7B, 7D-7E). Quantification of immunostained sections (normalized to total protein expression and highest fluorescent value obtained) showed a similar pattern to Western blotting with a $52 \%$ and $28 \%$ increase in $p$-AKT $/ t$-AKT and $p$-ERK $/ t$ ERK levels, respectively, in the CXCR4 ${ }^{+} \mathrm{TNBC}$ :CAFs model (Figure 7C, 7F). We note that high levels of ERK and $\mathrm{AKT}$ at the periphery of immunostained sections is due to the positioning of CXCR4 ${ }^{+} \mathrm{TNBC}$ cells at the periphery of the co-culture spheroids. Fibroblasts were mainly distributed in the center of the sections and showed minimal staining, clear from the composite image in Supplementary Figure 2 and Supplementary Movie 1. Therefore, quantification was only performed on the CXCR4 ${ }^{+}$TNBC cells of stained sections.

\section{Drug sensitivity of stromal-cancer cells co-culture spheroids}

Biochemical interactions of activated stromal cells and cancer cells are suggested as a major cause of drug resistance. To demonstrate the effect of CXCL12 -
CXCR4 signaling on drug response of breast cancer cells, we evaluated the sensitivity of co-culture spheroids to a standard chemotherapy drug, paclitaxel, to which TNBC patients may develop resistance [86-88]. Cancer cells in $\mathrm{CXCR}^{+} \mathrm{TNBC}: \mathrm{CAFs}$ co-culture spheroids were significantly more resistant to paclitaxel at a $0.1-10 \mathrm{nM}$ concentration range than cancer cells in the CXCR4 $4^{+}$TNBC:HMF spheroids $(p<0.01)$. The largest difference in cancer cell viability $(36 \%)$ occurred at a drug concentration of $1 \mathrm{nM}$ (Figure 8). This demonstrates the impact of activated tumor stromal cells on chemotherapy resistance of cancer cells, consistent with several other reports [89-92], and that our 3D tumor models recapitulate stromal-mediated drug resistance. Treatment with the CXCR4 antagonist AMD3100 eliminated resistance to paclitaxel of cancer cells in the CXCR4 ${ }^{+} \mathrm{TNBC}$ :CAFs model. This result emphasizes the potential to improve response to chemotherapy by targeting the CXCL12CXCR4 signaling [93-95].

To understand the mechanism of paclitaxel resistance, we performed IHC analysis of cryosections of spheroids to assess ERK and AKT activity in CXCR4 ${ }^{+}$TNBC:CAFs and CXCR4 ${ }^{+}$TNBC:HMF models treated with $1 \mathrm{nM}$ paclitaxel. Following treatment, significantly higher phosphorylation levels of both proteins were maintained in the $\mathrm{CXCR} 4^{+} \mathrm{TNBC}$ :CAFs model $(p<0.05)$, indicating that resistance to paclitaxel due to the CXCL12 - CXCR4 signaling is mediated by activation of these kinase pathways (Figure 9 and Supplementary Figure 3). Previous studies showed that paclitaxel resistance strongly correlated with increased activation of the MAPK and PI3K pathways in breast cancers [96-99], supporting our data. Upon co-treatment of spheroids with $1 \mu \mathrm{M}$ of AMD3100, phosphorylated levels of ERK and AKT in the CXCR4 ${ }^{+}$TNBC:CAFs model treated with paclitaxel normalized to that of control $\mathrm{CXCR} 4^{+} \mathrm{TNBC}: \mathrm{HMF}$ spheroids. These results collectively demonstrate the promising therapeutic approach of sensitizing TNBC
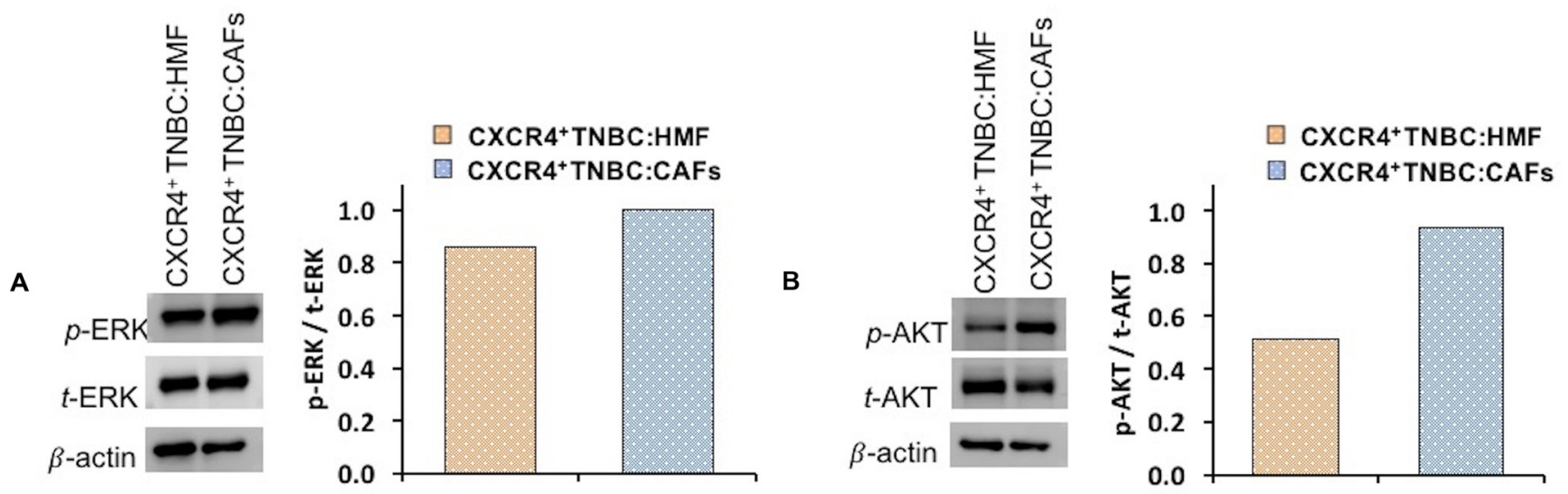

Figure 6: Western blot analysis of signaling proteins (A) $p$-ERK and (B) $p$-AKT in co-cultures of CXCR4 $4^{+}$TNBC cells with HMF and CAFs. Quantified data were normalized to $\beta$-actin protein expression. 
tumors to chemotherapy treatment by disrupting the cancer-stroma signaling and its downstream pathways to overcome stroma-induced cancer cell growth and chemotherapy resistance [100, 101].

Our findings suggest that greater proliferation and drug resistance of cancer cells in the CXCR4 ${ }^{+} \mathrm{TNBC}$ :CAFs model is due to CXCL12 - CXCR4 signaling through
AKT and ERK. Thus, we hypothesized that inhibition of these kinase pathways may block enhanced growth of cancer cells and compromise their viability. We treated both co-culture spheroids with specific inhibitors of MEK/ ERK (PD0325901) and PI3K/AKT (PI-103) at a wide concentration range for a six-day period. Effectiveness of the inhibitors was assessed by eGFP detection of breast
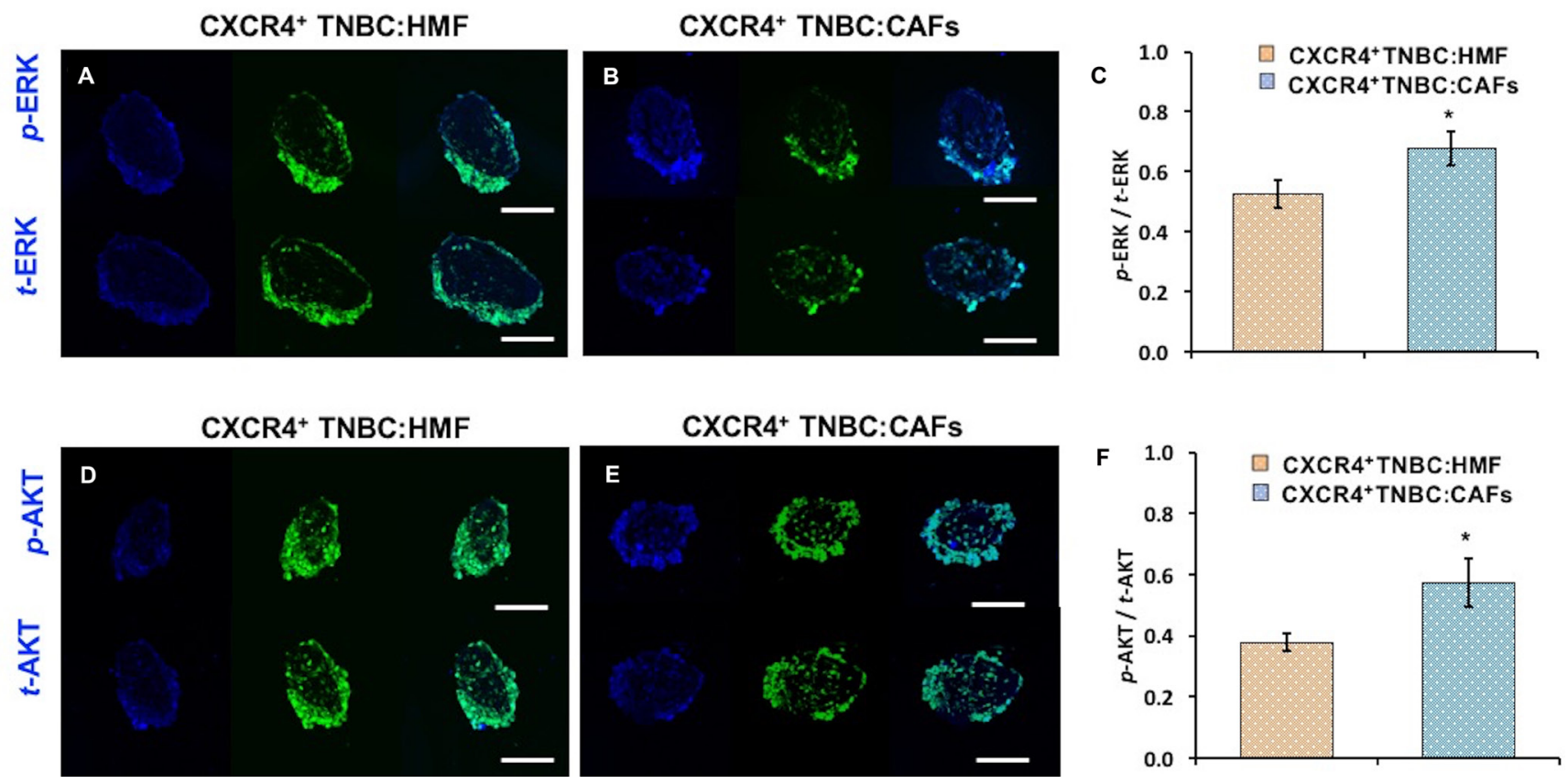

Figure 7: Cryosections of co-culture spheroids of CXCR4 ${ }^{+}$TNBC cells (green) with HMF and CAFs immunostained for (A-B) ERK and (D-E) AKT. A blue fluorescent secondary antibody was used to detect ERK and AKT. Images represent spheroids on day 4 of culture. Quantification showed higher (C) ERK and (F) AKT phosphorylation in the CXCR4 TNBC cells when co-cultured with CAFs. Scale bar is $200 \mu \mathrm{m} .{ }^{*} p<0.05$.
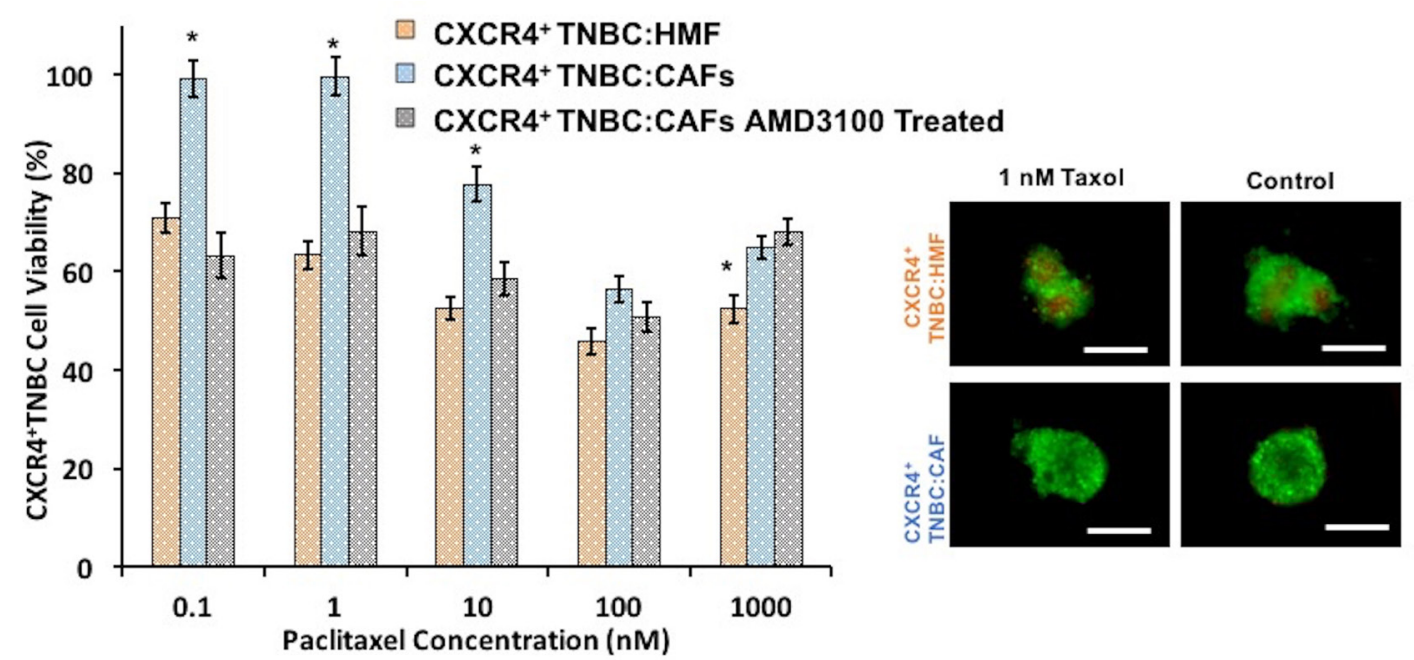

Figure 8: CXCR4 ${ }^{+} \mathrm{TNBC}: \mathrm{CAFs}$ co-culture spheroids (blue bars) display resistance to paclitaxel treatment but lose resistance to the drug when co-treated with a CXCR4 receptor antagonist AMD3100 (gray bars), similar to the negative control counterpart (CXCR4 ${ }^{+}$TNBC:HMF, orange bars). Images reflect spheroids at the end of drug treatment period (6 days). Scale bar is $200 \mu \mathrm{m} .{ }^{*} p<0.01$. 
cancer cells in treated groups relative to their respective negative control group (no treatment) and presented as percent cell viability. We found that breast cancer cells in the CXCR4 ${ }^{+}$TNBC:CAFs model were more sensitive to PD0325901 treatment below $100 \mathrm{nM}$ (Figure 10A) and PI-103 treatment below $1 \mu \mathrm{M}$ (Figure 10B). We further confirmed the reduction in breast cancer cell viability using quantification of fluorescence images of drug-treated spheroids in Figure 10C-10D. The largest differences in the viability of $\mathrm{CXCR} 4{ }^{+} \mathrm{TNBC}$ cells in co-culture with CAFs and HMF were $23 \%$ and $27 \%$ at $1 \mathrm{nM}$ of PD0325901 and $0.1 \mathrm{nM}$ of PI-103, respectively. Interestingly, PI-103 at a
$0.1 \mathrm{nM}$ concentration reduced breast cancer cell viability in the $\mathrm{CXCR} 4^{+} \mathrm{TNBC}$ :CAFs model to $56 \%$. This was consistent with protein expression results that showed significant activation of the PI3K/AKT pathway (Figure 6 and 7) leads to increased growth of the breast cancer cells (Figure 4). This also agrees with a study that showed CXCL12 signaling-mediated drug resistant cancer cells became substantially more sensitive to chemotherapy through AKT inhibition [90]. At higher concentrations, the MEK/ERK pathway inhibitor is more effective and reduces the cancer cells viability to less than $10 \%$. Overall, the results corroborate our protein expression study that

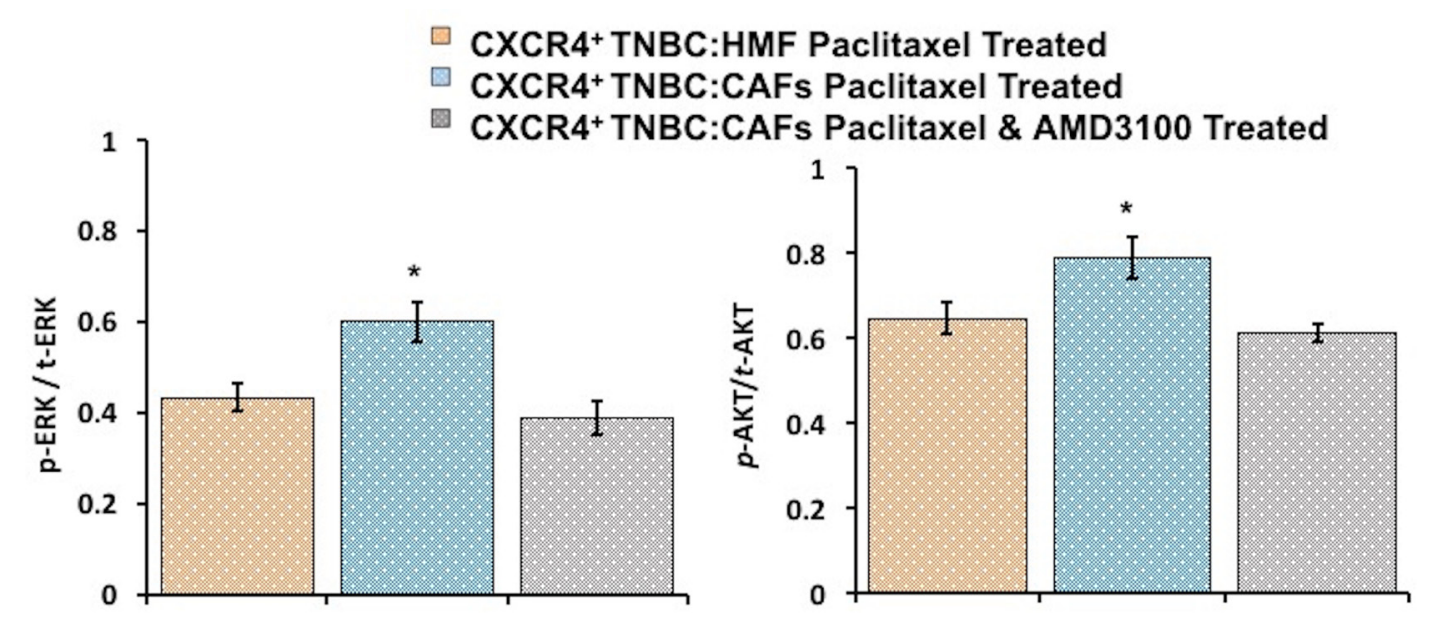

Figure 9: Immunohistochemical analysis of drug-treated spheroids indicates that increases in AKT and ERK phosphorylation levels in CXCR4 ${ }^{+}$TNBC:CAF co-culture spheroids contribute to paclitaxel resistance (blue bars). Co-treatment with a CXCR4 receptor antagonist, AMD3100, lowers AKT and ERK activities (gray bars) to that observed in the paclitaxel sensitive control culture (CXCR4 ${ }^{+}$TNBC:HMF, orange bars). ${ }^{*} p<0.05$.
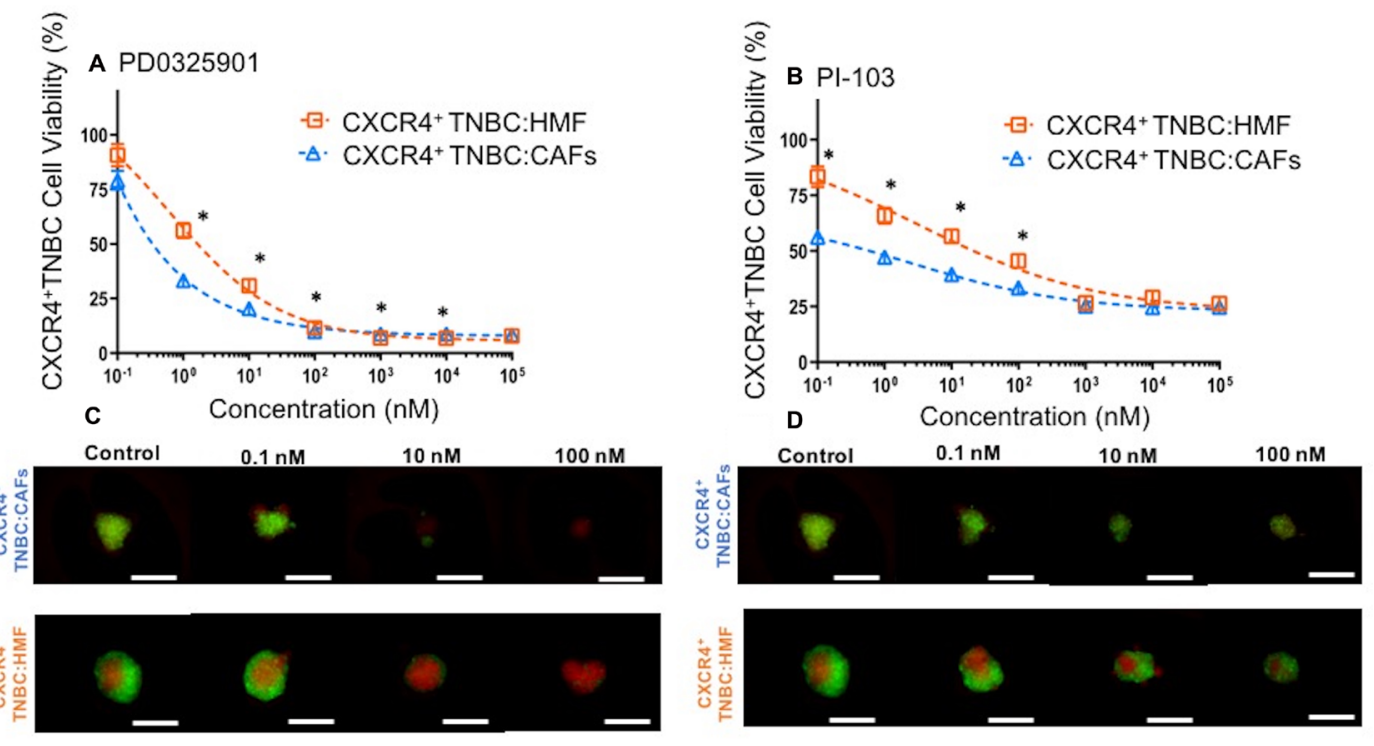

Figure 10: Viability of CXCR4 ${ }^{+}$TNBC cells in co-culture with HMF and CAFs treated with a MEK inhibitor, (A) PD0325901, and a PI3K inhibitor, (B) PI-103, measured using the endogenous eGFP signal of the TNBC cells. CXCR4 ${ }^{+}$TNBC cells in co-culture with CAFs show greater drug sensitivity to the molecular inhibitors. Images reflect spheroids at end of drug treatment period (5 days). Error bars represent standard error of mean. Scale bar is $200 \mu \mathrm{m} .{ }^{*} p<0.05$. 
showed upregulated activity of these two kinase pathways due to CXCL12 - CXCR4 signaling. Although CXCL12 CXCR4 can potentially activate multiple pathways in cancer cells [102], these results establish signaling to MAPK and PI3K pathways in TNBC cells and the promising result of targeting these pathways to improve cytotoxicity. Considering the activation of both kinase pathways due to this signaling, their simultaneous inhibition using combination treatments with specific molecular inhibitors of AKT and MAPK pathways may result in synergistic inhibition of TNBC cell growth and survival at lower drug concentrations than those with single-agent treatments [103]. Collectively, these results strongly support the utility of our 3D tumor models for cellular and molecular studies of tumor-stromal interactions.

In conclusion, our robotic technology allowed facile generation of co-culture spheroids to conveniently study stromal-cancer (TNBC) cells interactions. We found that CXCL12 from CAFs signaled through CXCR4 on triple negative cancer cells to significantly enhance TNBC cell proliferation. Additionally, CAFs showed greater proliferation than their normal HMF counterpart. Increased proliferation of TNBC cells occurred due to activation of MAPK and PI3K signaling pathways, two known effectors of CXCR4 signaling. CXCL12 - CXCR4 signaling also conferred resistance to chemotherapy. We demonstrated the potential to overcome stromal-mediated drug resistance using small molecule inhibitors of MAPK and PI3K pathways and CXCR4 signaling. Altogether, these findings highlight the power of our model system to identify promising new therapeutic strategies to improve response to therapy in TNBC. Future studies with improved tumor models should address and distinguish effects of stromal cells-deposited ECM signaling and stromal cells-mediated biochemical signaling with cancer cells on the proliferation and drug response of cancer cells.

\section{MATERIALS AND METHODS}

\section{Aqueous two-phase system (ATPS) preparation}

Two polymers, BioUltra polyethylene glycol (PEG) (Sigma), MW: $35 \mathrm{kDa}$, and dextran (DEX) (Pharmacosmos), MW: $500 \mathrm{kDa}$, were utilized to form an aqueous two-phase system, as described previously [38, 104]. Briefly, PEG and DEX were added to the complete cell culture medium at $5.0 \%(\mathrm{w} / \mathrm{v})$ and $12.8 \%(\mathrm{w} / \mathrm{v})$, respectively. The polymers were dissolved using a vortex mixer and then incubating in a water bath at $37^{\circ} \mathrm{C}$ for 1 hour. The polymer solutions were then stored at $4^{\circ} \mathrm{C}$ until use. Prior to use, the PEG phase polymer solution was filtered by passing it through a $0.2 \mu \mathrm{m}$ syringe filter to remove any impurities.

\section{Cell culture}

Three different triple negative breast cancer cells were utilized for experiments. The cell lines were: MDA-
MB-231 (labeled TNBC), MDA-MB-231 cells expressing the CXCR4 receptor (labeled CXCR4 ${ }^{+} \mathrm{TNBC}$ ), and MDAMB-231 cells expressing the CXCR7 receptor (labeled CXCR $7^{+}$TNBC). These cells were previously stably transduced using lentiviral vectors and the expression of CXCR4 and CXCR7 receptors on cancer cells was confirmed through flow cytometry [105]. The TNBC cells were additionally stably transduced to express eGFP for detection [105]. In addition, a human mammary fibroblast (HMF) cell line (labeled HMF) stably transduced with mCherry protein was utilized [106]. HMF cells were also stably transduced to secrete $0.75 \mathrm{ng} / \mathrm{ml} / \mathrm{hr}$ of CXCL12- $\alpha$ fused with mCherry for tracking, and the resulting cells were labeled as CAFs [56, 107]. CAFs extracted from breast cancer patients secreted varying amounts of CXCL12 in media ranging from $0.9 \mathrm{ng} / \mathrm{ml}-2 \mathrm{ng} / \mathrm{ml}$ over a $48 \mathrm{hr}$ time period, depending on the patients and the tumor samples [16]. The higher CXCL12 secretion rate of our transduced HMFs accounts for medium renewals and drug additions that consequently dilute the CXCL12 concentration in the medium. To maintain the cells in culture, they were grown in T175 flasks at $37^{\circ} \mathrm{C}$ and $5 \% \mathrm{CO}_{2}$ using Dulbecco's Modified Medium (DMEM) (Sigma) supplemented with 10\% fetal bovine serum (FBS) (Sigma), 1\% glutamine (Life Technologies), and $1 \%$ antibiotic (Life Technologies). Once a confluent monolayer formed, cells were rinsed with phosphate buffered saline (PBS) (Sigma) and dissociated using $5 \mathrm{ml}$ trypsin (Life Technologies) in an incubator for $\sim 2 \mathrm{~min}$. The cells were then neutralized with $10 \mathrm{ml}$ of the complete growth medium, collected, and centrifuged for $5 \mathrm{~min}$ at $1000 \mathrm{rpm}$. The supernatant was aspirated, cells were resupsended in $1 \mathrm{ml}$ of fresh medium, and loaded into a hemocytometer for counting.

\section{Co-culture spheroid formation using aqueous two-phase system (ATPS)}

A ratio of 1:2 TNBC to stromal cells was used to form co-culture spheroids that model the high stromal content often observed in larger and more advanced human breast tumors [5-8]. Our initial tests with 1:3 and 1:4 ratios (TNBC cells to fibroblasts) while keeping the initial TNBC cell density constant did not make any further changes in the growth of cancer cells. Therefore, the selected ratio of 1:2 (TNBC cells to fibroblasts) was used for all the studies. To prepare for forming spheroids, $30 \mu \mathrm{l}$ of the PEG phase solution was dispensed into each well of a 384-well plate, labeled as the destination plate. The DEX phase solution was mixed at an equal volume with a specific density of cells $\left(1 \times 10^{4} / 0.3 \mu \mathrm{l}\right.$ for monoculture spheroids of TNBC cells, $2 \times 10^{4} / 0.3 \mu \mathrm{l}$ for monocultures spheroids of $\mathrm{HMF}$ and CAFs, and $3 \times 10^{4} / 0.3 \mu$ for co-culture spheroids of TNBC-HMF and TNBC-CAFs). The DEX phase solution and the cell suspension became diluted in half upon mixing resulting in a final polymer concentration of $6.4 \%(\mathrm{w} / \mathrm{v})$, and densities of $5 \times 10^{3} / 0.3 \mu \mathrm{l}$ TNBC cells 
in monoculture spheroids, $1 \times 10^{4} / 0.3 \mu \mathrm{l}$ HMF cells or CAFs in monoculture spheroids, and $1.5 \times 10^{4} / 0.3 \mu \mathrm{l}$ in co-culture spheroids of TNBC-HMF cells or TNBCCAFs. A source 384-well plate was prepared by loading the wells from a single column with $\sim 20 \mu$ of the solution of mixed DEX phase and cell suspension. A robotic liquid handler (Bravo SRT, Agilent) was programmed to mix the source plate content and aspirate $0.3 \mu \mathrm{l}$ from each well. This solution was robotically dispensed as a single drop into each well of the destination plate containing the PEG phase. This process was repeated for all columns of the destination plate. After completion, the destination plate was placed in an incubator to allow cells to aggregate into a single spheroid in each well within 48 hrs. Our studies used a total of eight co-culture models: TNBC:HMF, TNBC:CAFs, CXCR4 ${ }^{+}$TNBC: HMF, CXCR4 ${ }^{+} \mathrm{TNBC}: \mathrm{CAFs}, \mathrm{CXCR} 7^{+} \mathrm{TNBC}: \mathrm{HMF}$, CXCR7 ${ }^{+} \mathrm{TNBC}: \mathrm{CAFs}, \mathrm{CXCR} 4^{+} / \mathrm{CXCR}^{+} \mathrm{TNBC}: \mathrm{HMF}$, and $\mathrm{CXCR}^{+} / \mathrm{CXCR}^{+} \mathrm{TNBC}$ :CAFs. Images of co-culture spheroids were captured using an inverted fluorescent microscope (Axio Observer, Zeiss) equipped with a highresolution camera (AxioCam MRm, Zeiss).

\section{Robotic media exchange of co-culture spheroids}

After forming spheroids, the cell culture medium in microwells was robotically exchanged to fresh medium containing only $\sim 1 \%$ FBS. This was to ensure minimizing the effect of serum on cell growth and capture the effect of paracrine stromal-cancer cells signaling [108, 109]. The medium exchange was performed by three consecutive robotic dispensing and aspirating of $40 \mu \mathrm{l}$ of the medium containing $1 \%$ FBS. This reduced the FBS concentration in the microwells from that of the complete growth medium to a very low level of $\sim 1 \%$. Therefore, the co-culture spheroids were maintained in $\sim 1 \%$ FBScontaining medium for the duration of experiments. The medium exchange also diluted the PEG and DEX polymer concentrations and resulted in a single medium phase. The ATPS was not necessary at this point as it was only used for spheroid formation. The liquid handling protocol was optimized to avoid loss of spheroids during the process of medium exchange. Following this step, spheroids remained in $70 \mu \mathrm{l}$ of homogenous $\sim 1 \%$ FBS-containing medium for the duration of culture and without any further medium exchange.

\section{Evaluation of metabolic activity of co-culture spheroids}

The growth of co-culture spheroids was evaluated for a six-day period for all eight co-culture models using a standard PrestoBlue metabolic activity assay (Invitrogen). The PrestoBlue reagent contains a resazurin compound that is reduced by metabolically active, viable cells to resorufin detectable with standard plate readers at excitation and emission wavelengths of 560 and 590 $\mathrm{nm}$, respectively [110]. We have previously optimized the PrestoBlue assay for use with spheroid cultures $[38,41]$. The resulting raw fluorescence data from all eight co-culture spheroids were normalized to the highest fluorescent readout obtained in the experimental data over the six-day period. Data from replicates $(n=14)$ of each co-culture model were then averaged and statistically evaluated to select certain co-culture models for further studies.

\section{Statistical analysis of growth of co-culture spheroids}

Statistical analysis was performed on the metabolic activity of co-culture models using a one-way multivariate analysis of variance (MANOVA). Briefly, the data represented measured signals from eight different coculture models over six days. For each model on each day, there were 14 experimental replicates that were repeatedly used over the six-day measurement period. Therefore, we selected one-way MANOVA for data analysis to account for the temporal dependency of the daily measurements with each spheroid from a given coculture model $[111,112]$. The data were tested in two steps: (i) to evaluate if a co-culture model was statistically different from the other models. This was done by testing the null hypothesis that none of the co-cultures was different from the other models; and (ii) to identify if the CXCR4 ${ }^{+}$TNBC:CAFs model was statistically different from the other seven models (TNBC:HMF, TNBC: CAFs, CXCR4 $4^{+} \mathrm{TNBC}: \mathrm{HMFs}, \mathrm{CXCR} 7^{+} \mathrm{TNBC}: \mathrm{HMF}$, CXCR $7^{+} \mathrm{TNBC}: \mathrm{CAFs}, \mathrm{CXCR} 4^{+} / \mathrm{CXCR} 7^{+} \mathrm{TNBC}: \mathrm{HMF}$, and $\mathrm{CXCR}^{+} / \mathrm{CXCR}^{+} \mathrm{TNBC}$ :CAFs). This was done using seven pairwise comparisons to test the null hypothesis that the CXCR4 ${ }^{+}$TNBC:CAFs co-culture model was not different from the other models.

The first statistical test showed significant differences among the eight co-culture models. Next, the CXCR4 ${ }^{+}$TNBC:CAFs model was selected for pairwise comparisons with the other seven models due to its higher levels of proliferation. We determined if greater proliferation in this model was statistically significant from the other co-cultures. The Bonferroni correction was utilized to control the family-wise error since this was a multiple testing problem [113]. A significance level of $\alpha$ $=0.05$ was selected to determine significant differences between the CXCR4 $4^{+}$TNBC:CAFs model and every other co-culture model. But $\alpha$ was first modified to $\alpha / m$, where $m$ accounts for the number of hypotheses made. In this analysis, the number of hypotheses was $m=7$. Therefore, a corrected significance level of 0.007 was used to reject the hypothesis that the CXCR4 $4^{+} \mathrm{TNBC}$ :CAFs model was not different from all the other seven co-cultures. 
For experiments that contained only two or three co-culture models presented in other sections, a student's $t$-test in Microsoft Excel Software or two-way ANOVA in Minitab Software was utilized with a $p<0.05$ representing statistical significance.

\section{Blocking and stimulating chemokine-receptor signaling in co-culture spheroids}

To further evaluate cell proliferation due to chemokine-receptor signaling in the $\mathrm{CXCR}^{+} \mathrm{TNBC}$ : $\mathrm{CAFs}$ co-culture spheroids, AMD3100 (Selleckchem) was used to block the signaling and CXCL12- $\alpha$ conditioned medium was used to stimulate the signaling. A stock solution of AMD3100 was prepared in distilled, sterile water at $5 \mathrm{mM}$ and stored at $-80^{\circ} \mathrm{C}$ according to the manufacturer's instructions. The desired concentration of AMD3100 was prepared by serially diluting the stock solution in $1 \%$ FBS-containing cell culture medium. After formation of spheroids in the PEG-DEX ATPS, the medium exchange was done by adding $40 \mu \mathrm{l}$ of AMD3 100 at a $1.75 \mu \mathrm{M}$ concentration to wells already containing 30 $\mu \mathrm{l}$ of medium. This diluted AMD3100 concentration to $1 \mu \mathrm{M}$. The AMD3100 solution was renewed on day four by removing $50 \mu \mathrm{l}$ of the well contents and adding $50 \mu \mathrm{l}$ of fresh solution of AMD3100 at a $1 \mu \mathrm{M}$ concentration.

To induce signaling in the CXCR4 ${ }^{+} \mathrm{TNBC}: \mathrm{HMF}$ co-culture spheroids, the spheroids were treated with CXCL12-containing conditioned medium collected from a confluent monolayer of CAFs. The conditioned medium was extracted from the CAFs monolayer 24 hours after seeding to ensure that it contained high levels of nutrients and growth factors. The conditioned medium was added to co-culture spheroids through medium exchange and renewed on day four by removing $50 \mu \mathrm{l}$ of the well contents and adding $50 \mu \mathrm{l}$ of fresh conditioned medium. Metabolic activity of co-culture spheroids following inhibition (AMD3100 treatment) or stimulation (CXCL12- $\alpha$ conditioned medium treatment) was evaluated daily using PrestoBlue over the six-day culture period. Data were compared to the respective negative controls, i.e., co-culture spheroids (CXCR4 ${ }^{+} \mathrm{TNBC}: \mathrm{HMF}$ for inhibition and $\mathrm{CXCR} 4^{+} \mathrm{TNBC}$ : $\mathrm{CAFs}$ for stimulation) that were grown in $70 \mu \mathrm{l}$ of $1 \%$ FBS-containing media without receiving any treatment. Daily measured raw data from the co-culture models over the six-day culture were normalized to the highest fluorescent value obtained and then used for statistical tests.

\section{TNBC cell growth in co-culture spheroids}

The growth of TNBC cells in the co-culture models (TNBC:HMF, TNBC:CAFs, CXCR4 ${ }^{+}$TNBC:HMF, CXCR4 $4^{+}$TNB:CAFs) that were selected based on the metabolic activity analysis was further confirmed by measuring the endogenous eGFP in TNBC cells using a plate reader at excitation and emission wavelengths of 485 and $530 \mathrm{~nm}$, respectively. The results were compared to those of the monoculture spheroids (TNBC and CXCR4 ${ }^{+}$TNBC). Each model had $n=14$ replicates. Data from each model were normalized to an arbitrary fluorescent value of 8000 a.u. for ease of comparison among different groups. Background fluorescence from the mCherry-expressing stromal cells in the co-culture spheroids was subtracted from the measured signal of TNBC cells. Fluorescent images were captured using a confocal microscope (Fluoview, FV1000, Olympus).

\section{Immunostaining and quantification of proliferation of co-culture spheroids}

To validate and quantify cell proliferation of co-culture spheroids, immunohistochemical analysis was performed using an established protocol [41, 114]. Co-culture spheroids were aspirated from microwells on day four and collected in $200 \mu \mathrm{l}$ microcentrifuge tubes. Medium used for transferring spheroids was carefully removed from the microcentrifuge tubes and $100 \mu \mathrm{l}$ of $4 \%$ formaldehyde was added to fix the spheroids for 10 minutes at room temperature. The spheroids were rinsed with $100 \mu \mathrm{l}$ of PBS three times for 5 minutes to remove the formaldehyde. A 30\% (w/v) sucrose solution was then added to remove water from spheroids and prevent crystal formation upon freezing. Once spheroids sank to the bottom of the microcentrifuge tubes, an equal volume $(100 \mu \mathrm{l})$ of tissue freezing medium (Triangle Biomedical Sciences) was added to each tube. Samples were kept at $4^{\circ} \mathrm{C}$ overnight. Frozen samples were prepared the following day by flash-freezing spheroids into biopsy-sized cryomolds with dry ice. The spheroids were embedded between two thin layers of tissue freezing medium while avoiding bubble formation. The frozen molds containing the spheroids were stored at $-80^{\circ} \mathrm{C}$ until use.

A cryostat (Leica CM 1850) was used to cryosection the spheroids to $10 \mu \mathrm{m}$-thick slices. Only the middle sections of spheroids were collected and assessed. Each slice was transferred onto Superfrost Plus microscopic slides (Fisher). The sections were stained using a standard immunostaining protocol with primary antibodies purchased from Cell Signaling including the proliferative cell marker Ki-67, phospho-Erk1/2 (phospho-p44/42 MAPK), total-Erk1/2 (p44/42 MAPK), phosphoAkt (Ser473), and total-Akt (pan). A blue fluorescent secondary antibody (AMCA-conjugated goat anti-rabbit, Jackson ImmunoResearch) was used for detection to avoid interfering with the endogenous mCherry and eGFP signals in the stromal and TNBC cells, respectively. The endogenous eGFP fluorescence of TNBC cells was used to distinguish TNBC and stromal cells. An inverted fluorescent microscope (Axio Observer, Zeiss) was used to capture fluorescent images. Image processing and analysis 
were performed in ImageJ Software (NIH) to quantify expression of each blue-labeled protein. The TNBC cells of sections were found through binary conversion of the green channel images to select and outline only the eGFPexpressing TNBC cells. This TNBC cell outline was then overlaid on the blue channel images to measure the mean gray value of protein-expressing TNBC cells. For Ki-67 analysis, this value was then subtracted from the total mean gray value of blue channel images to determine the mean gray value of $\mathrm{Ki}-67^{+}$stromal cells.

\section{Western blot analysis with spheroids}

Co-culture spheroids (CXCR4 ${ }^{+} \mathrm{TNBC}$ :CAFs and CXCR4 ${ }^{+}$TNBC:HMF) were harvested from microplates on day four and transferred into $50 \mathrm{~mL}$ conical tubes. After centrifuging down the samples, the supernatant was removed and spheroids were washed with PBS. Spheroids were lysed using $500 \mu \mathrm{l}$ of complete RIPA buffer (50 mM tric-HCL, $150 \mathrm{mM} \mathrm{NaCl} \mathrm{1 \%} \mathrm{NP-40,} \mathrm{0.5 \%}$ sodium deoxycholate, and 0.1\% SDS, pH $7.4 \pm 0.2$ ), a protease inhibitor (complete mini, Roche Diagnostics), and phosphatase inhibitor (Life Technologies). Spheroids were sonicated (Vibra-Cell, Sonics) twice for five seconds at a 50\% amplitude level to ensure complete lysis. A BCA quantification assay kit (Life Technologies) was then used to quantify the total protein concentration extracted from spheroids. Electrophoresis and electroblotting were performed by protein addition $(20 \mu \mathrm{l})$ onto a $4-15 \%$ gel (Biorad) and then gel transferring onto a nitrocellulose membrane, respectively. The membranes were blocked with 5\% BSA (Sigma) for 1 hour. Primary antibodies purchased from Cell Signaling for phospho-Erk1/2 (phospho-p44/42 MAPK), total-Erk1/2 (p44/42 MAPK), phospho-Akt (Ser473), and total-Akt (pan) were prepared at concentrations recommended by the manufacturer, and incubated with membranes overnight at $4^{\circ} \mathrm{C}$. The membranes were then incubated with a horseradish peroxidase (HRP)-conjugated secondary antibody for 1 hour. The membranes were thoroughly washed prior to and following secondary antibody treatment and detected with an ECL chemiluminescence detection kit (GE Healthcare) using a FluorChem E imaging system (ProteinSimple). Quantified protein expression data were normalized to their corresponding $\beta$-actin expression.

\section{Chemotherapy drug testing with co-culture spheroids}

Both CXCR4 ${ }^{+}$TNBC:CAFs and CXCR4 ${ }^{+}$TNBC:HMF spheroids were subjected to (i) single treatment with a standard chemotherapy drug, paclitaxel (Selleckchem), and (ii) co-treatment with paclitaxel and AMD3100, to evaluate drug responses of TNBC cells. A stock solution of paclitaxel was prepared in $\mathrm{DMSO}$ at a $23 \mathrm{mM}$ concentration and stored at $-80^{\circ} \mathrm{C}$.
For single drug treatment, paclitaxel drug solutions were prepared in $1 \%$ FBS-containing cell culture medium at $2 \mathrm{X}$ the desired concentration. The drug solutions were added to co-culture spheroids, resulting in a total media volume of $70 \mu \mathrm{l}$ and diluting the drug concentration in half. For co-treatment of spheroids with paclitaxel and AMD3100, drug solutions were prepared in $1 \%$ FBS-containing cell culture medium at $3.5 \mathrm{X}$ the desired concentration and directly added (20 $\mu \mathrm{l}$ of each drug solution) to co-culture spheroids in wells already containing $30 \mu \mathrm{l}$ of media. Paclitaxel was added at a wide range of concentrations $(0.01-1000 \mathrm{nM})$ while AMD3100 was used at a constant $1 \mu \mathrm{M}$ concentration for co-treatments. Single-agent (paclitaxel) and co-treatment (paclitaxel and AMD3100) tests were conducted for six days. A small volume $(5 \mu \mathrm{l})$ of concentrated AMD3100 $(15 \mu \mathrm{M})$ was directly added to the co-treated spheroid cultures after 72 hrs of incubation to provide a fresh dose of $1 \mu \mathrm{M}$ AMD3100. Each condition in drug treatment studies used 14 spheroids. After six days, the TNBC cell viability of drug treated co-culture spheroids was evaluated using their endogenous eGFP fluorescence. Fluorescent readouts from treatments were normalized to the respective negative control conditions (no treatment) to calculate $\mathrm{CXCR} 4^{+} \mathrm{TNBC}$ cell viability.

\section{Preparation of inhibitors and testing against co-culture spheroids}

The CXCR4 ${ }^{+} \mathrm{TNBC}: \mathrm{HMF}$ and $\mathrm{CXCR} 4^{+} \mathrm{TNBC}$ : CAFs spheroids were treated with a MEK inhibitor, PD0325901 (Selleckchem), and a PI3K inhibitor, PI103 (Selleckchem). Stock solutions of the inhibitors were prepared at a $50 \mathrm{mM}$ concentration in DMSO and stored at $-80^{\circ} \mathrm{C}$. The desired inhibitor concentrations for experiments were prepared by serially diluting each stock solution in $1 \% \mathrm{FBS}$-containing cell culture medium. After forming co-culture spheroids, the medium exchange was performed as previously described and $30 \mu \mathrm{l}$ of an inhibitor solution (prepared at $2 \mathrm{X}$ desired concentration) was added to spheroids in the last renewal stage, diluting the inhibitor concentrations in half. A column of spheroids was left untreated and only received $30 \mu \mathrm{l}$ of fresh $1 \%$ FBS-containing medium. Spheroids were incubated for 72 hrs and then treatments were renewed by direct addition of $30 \mu \mathrm{l}$ of fresh inhibitor solutions (prepared at $1 \mathrm{X}$ desired concentration). The negative control conditions received 1\% FBS-containing medium. The spheroids were incubated for an additional $48 \mathrm{hrs}$ and viable TNBC cells in co-culture spheroids were detected using their endogenous eGFP with a plate reader. Fluorescent readouts from treatments were normalized to their respective negative controls (no treatment) to calculate CXCR4 ${ }^{+}$TNBC cell viability. The results obtained from plate reading were further confirmed by fluorescent microscopy (Axio Observer, Zeiss) followed by image analysis (Image J, NIH). 


\section{CONFLICTS OF INTEREST}

Authors declare no conflict of interest.

\section{FUNDING}

Funding for this work was provided by grants from United States National Institutes of Health grants R01CA196018, R01CA195655, U01CA210152, and R21CA182333, and National Science Foundation 1264562.

\section{REFERENCES}

1. Bhowmick NA, Neilson EG, Moses HL. Stromal fibroblasts in cancer initiation and progression. Nature. 2004; 432:332-7.

2. Hanahan D, Weinberg RA. Hallmarks of Cancer: The Next Generation. Cell. 2011; 144:646-74.

3. Balkwill FR, Capasso M, Hagemann T. The tumor microenvironment at a glance. J Cell Sci. 2012; 125:5591-6.

4. Shekhar MP, Pauley R, Heppner G. Extracellular matrix - stromal cell contribution to neoplastic phenotype of epithelial cells in the breast. Breast Cancer Res. 2003; 5.

5. de KruijfEM, van Nes JG, van de Velde CJ, Putter H, Smit VT, Liefers GJ, Kuppen PJ, Tollenaar RA, Mesker WE. Tumor-stroma ratio in the primary tumor is a prognostic factor in early breast cancer patients, especially in triplenegative carcinoma patients. Breast Cancer Res Treat. 2011; 125:687-96.

6. Moorman AM, Vink R, Heijmans HJ, van der Palen J, Kouwenhoven EA. The prognostic value of tumour-stroma ratio in triple-negative breast cancer. Eur J Surg Oncol. 2012; 38:307-13.

7. Dekker TJ, van de Velde CJ, van Pelt GW, Kroep JR, Julien JP, Smit VT, Tollenaar RA, Mesker WE. Prognostic significance of the tumor-stroma ratio: validation study in node-negative premenopausal breast cancer patients from the EORTC perioperative chemotherapy (POP) trial (10854). Breast Cancer Res Treat. 2013; 139:371-9.

8. Gujam FJA, Edwards J, Mohammed ZMA, Going JJ, Mcmillan DC. The relationship between the tumour stroma percentage, clinicopathological characteristics and outcome in patients with operable ductal breast cancer. Br J Cancer. Nature Publishing Group. 2014; 111:157-65.

9. Orimo A, Weinberg RA. Stromal Fibroblasts in Cancer: A Novel Tumor-Promoting Cell Type. Cell Cycle. 2006; 5:1597-601.

10. Kalluri R. The biology and function of fibroblasts in cancer. Nat Rev Cancer. 2016; 16:582-98.

11. Pinto MP, Dye WW, Jacobsen BM, Horwitz KB. Malignant stroma increases luminal breast cancer cell proliferation and angiogenesis through platelet-derived growth factor signaling. BMC Cancer. 2014; 14:1-12.
12. Seip K, Fleten KG, Barkovskaya A, Nygaard V, Haugen $\mathrm{MH}$, Engesaeter BO, Maelandsmo GM, Prasmickaite L. Fibroblast-induced switching to the mesenchymal-like phenotype and PI3K/mTOR signaling protects melanoma cells from BRAF inhibitors. Oncotarget. 2016; 7:1999720015. https://doi.org/10.18632/oncotarget.7671.

13. Stuelten CH, Byfield SD, Arany PR, Karpova TS, Stetlerstevenson WG, Roberts AB. Breast cancer cells induce stromal fibroblasts to express MMP-9 via secretion of TNF$\alpha$ and TGF- $\beta$. J Cell Sci. 2005; 118:2143-53.

14. Cirri P, Chiarugi P. Cancer associated fibroblasts: the dark side of the coin. Am J Cancer Res. 2011; 1:482-97.

15. Sappnio A, Skalli O, Jackson B, Schurch W, Gabbiani G. Smooth-muscle differentiation in stromal cells of malignant and non-malignant breast tissues. Int J Cancer. 1988; 41:701-12.

16. Orimo A, Gupta PB, Sgroi DC, Arenzana-Seisdedos F, Delaunay T, Naeem R, Carey VJ, Richardson AL, Weinberg RA. Stromal Fibroblasts Present in Invasive Human Breast Carcinomas Promote Tumor Growth and Angiogenesis through Elevated SDF-1/CXCL12 Secretion. Cell. 2005; 121:335-48.

17. Sun X, Cheng G, Hao M, Zheng J, Zhou X, Zhang J, Taichman RS, Pienta KJ, Wang J. CXCL12/CXCR4/ CXCR7 Chemokine Axis and Cancer Progression. Cancer Mestastasis Rev. 2010; 29:709-22.

18. Duda DG, Kozin SV, Kirkpatrick ND, Xu L, Fukumura D, Jain RK. CXCL12 (SDF1 $\alpha$ )-CXCR4/CXCR7 pathway inhibition: An emerging sensitizer for anticancer therapies? Clin Cancer Res. 2011; 17:2074-80.

19. Jiang Y, Wu X, Shi B, Wu W, Yin G. Expression of chemokine CXCL12 and its receptor CXCR4 in human epithelial ovarian cancer: An independent prognostic factor for tumor progression. Gynecol Oncol. 2006; 103:226-33.

20. Sun Y, Mao X, Fan C, Liu C, Guo A, Guan S, Jin Q, Li B, Yao F, Jin F. CXCL12-CXCR4 axis promotes the natural selection of breast cancer cell metastasis. Tumor Biol. 2014; 35:7765-73.

21. Luker KE, Luker GD. Functions of CXCL12 and CXCR4 in breast cancer. Cancer Lett. 2006; 238:30-41.

22. The Cancer Genome Atlas Network. Comprehensive molecular portraits of human breast tumours. Nature. 2012; 490:61-70.

23. Zhao S, Chang SL, Linderman JJ, Feng FY, Luker GD. A Comprehensive Analysis of CXCL12 Isoforms in Breast Cancer. Transl Oncol Neoplasia. 2014; 7:429-38.

24. Chu QD, Panu L, Holm NT, Li BDL, Johnson LW, Zhang S. High Chemokine Receptor CXCR4 Level in Triple Negative Breast Cancer Specimens Predicts Poor Clinical Outcome. J Surg Res. 2010; 159:689-95.

25. Bianchini G, Balko JM, Mayer IA, Sanders ME, Gianni L. Triple-negative breast cancer: challenges and opportunities of a heterogeneous disease. Nat Rev Clin Oncol. 2016; 13:674-90. 
26. Kunz-Schughart LA. Multicellular tumor spheroids: intermediates between monolayer culture and in vivo tumor. Cell Biol Int. 1999; 23:157-61.

27. Friedrich J, Ebner R, Kunz-Schughart LA. Experimental anti-tumor therapy in 3-D: spheroids--old hat or new challenge? Int J Radiat Biol 83: 849-71.

28. Kim JW, Ho WJ, Wu BM. The role of the 3D environment in hypoxia-induced drug and apoptosis resistance. Anticancer Res. 2011; 31:3237-45.

29. Herter-Sprie GS, Kung AL, Wong K. New cast for a new era: preclinical cancer drug development revisited. J Clin Invest. 2013; 123:3639-45.

30. Bradford JR, Wappett M, Beran G, Logie A, Delpuech O, Brown H, Boros J, Camp NJ, McEwen R, Mazzola AM, D'Cruz C, Barry ST. Whole transcriptome profiling of patient-derived xenograft models as a tool to identify both tumor and stromal specific biomarkers. Oncotarget. 2016; 7:20773-87. https://doi.org/10.18632/oncotarget.8014.

31. Yang Z, Xu S, Jin P, Yang X, Li X, Wan D, Zhang T, Long S, Wei X, Chen G, Meng L, Liu D, Fang Y, et al. MARCKS contributes to stromal cancer-associated fibroblast activation and facilitates ovarian cancer metastasis. Oncotarget. 2016; 7:37649-63. https://doi.org/ 10.18632/oncotarget.8726.

32. Kononen J, Bubendorf L, Kallioniemi A, Barlund M, Schraml P, Leighton S, Torhorst J, Mihatsch M, Sauter G, Kallioniemi O. Tissue microarrays for high-throughput molecular profiling of tumor specimens. Nat Med. 1998; 4:844-7.

33. Voduc D, Kenney C, Nielsen TO. Tissue Microarrays in Clinical Oncology. Semin Radiat Oncol. 2008; 18:89-97.

34. McCuaig R, Wu F, Dunn J, Rao S, Dahlstrom JE. The biological and clinical significance of stromal-epithelial interactions in breast cancer. Pathology. 2017; 49:133-40.

35. Lee GH, Lee JS, Wang X, Lee SH. Bottom-Up Engineering of Well-Defined 3D Microtissues Using Microplatforms and Biomedical Applications. Adv Healthc Mater. 2016; 5:56-74.

36. Ham SL, Joshi R, Thakuri PS, Tavana H. Liquid-based three-dimensional tumor models for cancer research and drug discovery. Exp Biol Med (Maywood) 2016; 241: 939-54.

37. Nyga A, Cheema U, Loizidou M. 3D tumour models: novel in vitro approaches to cancer studies. J Cell Commun Signal. 2011; 5:239-48.

38. Lemmo S, Atefi E, Luker GD, Tavana H. Optimization of Aqueous Biphasic Tumor Spheroid Microtechnology for Anti-cancer Drug Testing in 3D Culture. Cell Mol Bioeng. 2014; 7:344-54.

39. Atefi E, Lemmo S, Fyffe D, Luker GD, Tavana H. High Throughput, Polymeric Aqueous Two-Phase Printing of Tumor Spheroids. Adv Funct Mater. 2014; 24:6509-15.

40. Thakuri PS, Ham SL, Luker GD, Tavana H. Multiparametric Analysis of Oncology Drug Screening with Aqueous TwoPhase Tumor Spheroids. Mol Pharm. 2016; 13: 3724-35.
41. Ham SL, Joshi R, Luker GD, Tavana H. Engineered Breast Cancer Cell Spheroids Reproduce Biologic Properties of Solid Tumors. Adv Healthc Mater. 2016; 5:2788-98.

42. Atefi E, Joshi R, Mann JA, Tavana H. Interfacial Tension Effect on Cell Partition in Aqueous Two-Phase Systems. ACS Appl Mater Interfaces. 2015; 7:21305-14.

43. Atefi E, Mann JA, Tavana H. Ultralow interfacial tensions of aqueous two-phase systems measured using drop shape. Langmuir. 2014; 30:9691-9.

44. Ham SL, Atefi E, Fyffe D, Tavana H. Robotic Production of Cancer Cell Spheroids with an Aqueous Two-phase System for Drug Testing. J Vis Exp. 2015; e52754.

45. Camp JT, Elloumi F, Roman-perez E, Rein J, Stewart DA, Harrell JC, Perou CM, Troester MA. Interactions with Fibroblasts Are Distinct in Basal-Like and Luminal Breast Cancers. Mol Cancer Res. 2011; 1:3-14.

46. Pietras K, Östman A. Hallmarks of cancer: Interactions with the tumor stroma. Exp Cell Res. 2010; 316:1324-31.

47. Sadlonova A, Novak Z, Johnson MR, Bowe DB, Gault SR, Page GP, Thottassery JV, Welch DR, Frost AR. Breast fibroblasts modulate epithelial cell proliferation in threedimensional in vitro co-culture. Breast Cancer Res. 2005; 7:R46-59.

48. Gache C, Berthois Y, Martin P, Saez S. Positive Regulation of Normal and Tumoral Mammary Epithelial Cell Proliferation by Fibroblasts in Coculture. Vitr Cell Dev Biol Anim.1998; 34: 347-51.

49. Dewan MZ, Ahmed S, Iwasaki Y, Ohba K, Toi M, Yamamoto N. Stromal cell-derived factor-1 and CXCR4 receptor interaction in tumor growth and metastasis of breast cancer. Biomed Pharmacother. 2006; 60:273-6.

50. Smith MCP, Luker KE, Garbow JR, Prior JL, Jackson E, Piwnica-worms D, Luker GD. CXCR4 Regulates Growth of Both Primary and Metastatic Breast Cancer. Cancer Res. 2004; 3100:8604-12.

51. Liang Z, Wu T, Lou H, Yu X, Taichman RS, Lau SK, Nie S, Umbreit J, Shim H. Inhibition of Breast Cancer Metastasis by Selective Synthetic Polypeptide against CXCR4. Cancer Res. 2004; 64:4302-8.

52. Liang Z, Yoon Y, Votaw J, Goodman MM, Williams L. Silencing of CXCR4 Blocks Breast Cancer Metastasis. Cancer Res. 2005; 65:967-72.

53. Epstein RJ. The CXCL12-CXCR4 chemotactic pathway as a target of adjuvant breast cancer therapies. Nat Rev Cancer. 2004; 4:901-9.

54. Naumann U, Cameroni E, Pruenster M, Mahabaleshwar H, Raz E, Rot A, Thelen M. CXCR7 Functions as a Scavenger for CXCL12 and CXCL11. PLoS One. 2010; 5:e9175.

55. Sanchez-Martin L, Sanchez-Mateos P, Cabanas C. CXCR7 impact on CXCL12 biology and disease. Cell. 2013; 19:12-22.

56. Luker KE, Lewin SA, Mihalko LA, Schmidt BT, Winkler JS, Coggins NL, Thomas DG, Luker GD. Scavenging of CXCL12 by CXCR7 Promotes Tumor Growth and 
Metastasis of CXCR4-positive Breast Cancer Cells. Oncogene. 2012; 31:4750-8.

57. Decaillot FM, Kazmi MA, Lin Y, Ray-Saha S, Sakmar TP, Sachdev P. CXCR7/CXCR4 Heterodimer Constitutively Recruits B-Arrestin to Enhance Cell Migration. J Biol Chem. 2011; 286:32188-97.

58. Rajagopal S, Kim J, Ahn S, Craig S, Lam CM, Gerard NP, Gerard C, Lefkowitz RJ. $\beta$-arrestin- but not G proteinmediated signaling by the "decoy" receptor CXCR7. Proc Natl Acad Sci. 2010; 107:628-32.

59. Thelen M, Thelen S. CXCR7, CXCR4 and CXCL12: An eccentric trio? J Neuroimmunol. 2008; 198:9-13.

60. Zabel BA, Wang Y, Lewén S, Berahovich RD, Penfold MET, Zhang P, Powers J, Summers BC, Miao Z, Zhao B, Jalili A, Janowska-Wieczorek A, Jaen JC, et al. Elucidation of CXCR7-Mediated Signaling Events and Inhibition of CXCR4-Mediated Tumor Cell Transendothelial Migration by CXCR7 Ligands. J Immunol. 2009; 183:3204-11.

61. Hernandez L, Magalhaes MA, Coniglio SJ, Condeelis JS, Segall JE. Opposing roles of CXCR4 and CXCR7 in breast cancer metastasis. Breast Cancer Res. 2011; 13:R128.

62. Wang J, Shiozawa Y, Wang J, Wang Y, Jung Y, Pienta KJ, Mehra R, Loberg R, Taichman RS. The Role of CXCR7/ RDC1 as a Chemokine Receptor for CXCL12/SDF-1 in Prostate Cancer. J Biol Chem. 2008; 283:4283-94.

63. Miao Z, Luker KE, Summers BC, Berahovich R, Bhojani MS, Rehemtulla A, Kleer CG, Essner JJ, Nasevicius A, Luker GD, Howard MC, Schall TJ. CXCR7 (RDC1) promotes breast and lung tumor growth in vivo and is expressed on tumor-associated vasculature. Proc Natl Acad Sci. 2007; 104:15735-40.

64. Smith MCP, Luker KE, Garbow JR, Prior JL, Jackson E, Piwnica-worms D, Luker GD. CXCR4 Regulates Growth of Both Primary and Metastatic Breast Cancer. Cancer Res. 2004; 64:8604-12.

65. Li J, Yu L, Shen Y, Zhou L, Wang Y, Zhang J. Inhibition of CXCR4 activity with AMD3100 decreases invasion of human colorectal cancer cells in vitro. World J Gastroenterol. 2008; 14:2308-13.

66. Marchesi F, Monti P, Leone BE, Zerbi A, Vecchi A, Piemonti L, Mantovani A, Allavena P. Increased Survival, Proliferation, and Migration in Metastatic Human Pancreatic Tumor Cells Expressing Functional CXCR4. Cancer Res. 2004; 64:8420-7.

67. Scotton CJ, Wilson JL, Scott K, Stamp G, Wilbanks GD, Fricker S, Bridger G, Balkwill FR. Multiple Actions of the Chemokine CXCL12 on Epithelial Tumor Cells in Human Ovarian Cancer. Cancer Res. 2002; 62:5930-8.

68. Lohr M, Schmidt C, Ringel J, Kluth M, Muller P, Nizze $\mathrm{H}$, Jesnowski R. Transforming Growth Factor- $\beta 1$ Induces Desmoplasia in an Experimental Model of Human Pancreatic Carcinoma. Cancer Res. 2001; 61:550-5.

69. Aoyagi Y, Oda T, Kinoshita T, Nakahashi C, Hasebe T, Ohkohchi N, Ochiai A. Overexpression of TGF-B by infiltrated granulocytes correlates with the expression of collagen mRNA in pancreatic cancer. Br J Cancer. 2004; 91:1316-26.

70. Kuperwasser C, Chavarria T, Wu M, Magrane G, Gray JW, Carey L, Richardson A, Weinberg RA. Reconstruction of functionally normal and malignant human breast tissues in mice. Proc Natl Acad Sci. 2004; 101:4966-71.

71. Olumi AF, Grossfeld GD, Hayward SW, Carroll PR, Tlsty TD, Cunha GR. Carcinoma-associated Fibroblasts Direct Tumor Progression of Initiated Human. Cancer Res. 1999; 59:5002-11.

72. Chatterjee S, Azad BB, Nimmagadda S. The Intricate Role of CXCR4 in Cancer. Adv Cancer Res. 2014; 124:31-43.

73. Rama-Esendagli D, Esendagli G, Yilmaz G, Guc D. Spheroid formation and invasion capacity are differentially influenced by co-cultures of fibroblast and macrophage cells in breast cancer. Mol Biol Rep. 2014; 41:2885-92.

74. Kunz-Schughart LA, Heyder P, Schroeder J, Knuechel R. A heterologous 3-D coculture model of breast tumor cells and fibroblasts to study tumor-associated fibroblast differentiation. Exp Cell Res. 2001; 266:74-86.

75. Steinberg MS, Takeichit M. Experimental specification of cell sorting, tissue spreading, and specific spatial patterning by quantitative differences in cadherin expression. Proc Natl Acad Sci. 1994; 91:206-9.

76. Madar S, Goldstein I, Rotter V. "Cancer associated fibroblasts" - more than meets the eye. Trends Mol Med. 2013; 19:447-53.

77. Weiswald LB, Bellet D, Dangles-Marie V. Spherical Cancer Models in Tumor Biology. Neoplasia. 2015; 17:1-15.

78. Erez N, Truitt M, Olson P, Hanahan D. Cancer-Associated Fibroblasts Are Activated in Incipient Neoplasia to Orchestrate Tumor-Promoting Inflammation in an NFkB-Dependent Manner. Cancer Cell. 2010; 17:135-47.

79. Wu J, Liang C, Chen M, Su W. Association between tumor-stroma ratio and prognosis in solid tumor patients: a systematic review and meta-analysis. Oncotarget. 2016; 7:68954-68965. https://doi.org/10.18632/oncotarget.12135.

80. Downey CL, Simpkins SA, White J, Holliday DL, Jones JL, Jordan LB, Kulka J, Pollock S, Rajan SS, Thygesen HH, Hanby AM, Speirs V. The prognostic significance of tumourstroma ratio in oestrogen receptor-positive breast cancer. $\mathrm{Br}$ J Cancer. 2014; 110:1744-7.

81. Luker KE, Luker GD. Functions of CXCL12 and CXCR4 in breast cancer. Cancer Lett. 2006; 238:30-41.

82. Sobolik T, Su YJ, Wells S, Ayers GD, Cook RS, Richmond A. CXCR4 drives the metastatic phenotype in breast cancer through induction of CXCR2 and activation of MEK and PI3K pathways. Mol Biol Cell. 2014; 25:566-82.

83. Cojoc M, Peitzsch C, Trautmann F, Polishchuck L, Telegeev GD, Dubrovska A. Emerging targets in cancer management: role of the CXCL12/CXCR4 axis. Onco Targets Ther. 2013; 6:1347-61. 
84. Hoeflich KP, O'Brien C, Boyd Z, Cavet G, Guerrero S, Jung K, Januario T, Savage H, Punnoose E, Truong T, Zhou $\mathrm{W}$, Berry L, Murray L, et al. In vivo Antitumor Activity of MEK and Phosphatidylinositol 3-Kinase Inhibitors in Basal-Like Breast Cancer Models. Clin Cancer Res. 2009; 15:4649-64.

85. Yee K, Weaver V, Hammer D. Integrin-mediated signalling through the MAP-kinase pathway. IET Syst Biol. 2008; 2:8-15.

86. André F, Zielinski CC. Optimal strategies for the treatment of metastatic triple-negative breast cancer with currently approved agents. Ann Oncol. 2012; 23:vi46-51.

87. Blum JL, Dieras V, Lo Russo PM, Horton J, Rutman O, Buzdar A, Osterwalder B. Multicenter, Phase II study of capecitabine in taxane-pretreated metastatic breast carcinoma patients. Cancer. 2001; 92:1759-68.

88. Rivera E, Gomez H. Chemotherapy resistance in metastatic breast cancer: the evolving role of ixabepilone. Breast Cancer Res. 2010; 12:S2.

89. Farmer P, Bonnefoi H, Anderle P, Cameron D, Wirapati P, Becette V, Andre S, Piccart M, Campone M, Brain E, MacGrogan G, Petit T, Jassem J, et al. A stroma-related gene signature predicts resistance to neoadjuvant chemotherapy in breast cancer. Nat Med. 2009; 15:68-74.

90. Singh S, Srivastava SK, Bhardwaj A, Owen LB, Singh AP. CXCL12 - CXCR4 signalling axis confers gemcitabine resistance to pancreatic cancer cells: a novel target for therapy. Br J Cancer. 2010; 103:1671-9.

91. Vianello F, Villanova F, Tisato V, Lymperi S, Ho K, Gomes AR, Marin D, Bonnet D, Apperley J, Lam EW, Dazzi F. Bone marrow mesenchymal stromal cells nonselectively protect chronic myeloid leukemia cells from imatinib-induced apoptosis via the CXCR4/CXCL12 axis. Haematologica. 2010; 95:1081-9.

92. Hartmann TN, Burger JA, Glodek A, Fujii N, Burger M. CXCR4 chemokine receptor and integrin signaling co-operate in mediating adhesion and chemoresistance in small cell lung cancer (SCLC) cells. Oncogene. 2005; 24:4462-71.

93. Domanska UM, Timmer-Bosscha H, Nagengast WB, Munnink THO, Kliphuis NM, Huls G, De Vries EGE, de Jong IJ, Walenkamp AME. CXCR4 Inhibition with AMD3100 Sensitizes Prostate Cancer to Docetaxel Chemotherapy. Neoplasia. 2012; 14:709-18.

94. Yu M, Gang EJ, Parameswaran R, Stoddart S, Fei F, Schmidhuber S, Park E, Hsieh YT, Yang AS, Groffen J, Heisterkamp N, Kim YM. AMD3100 sensitizes acute lymphoblastic leukemia cells to chemotherapy in vivo. Blood Cancer J. 2011; 1:e14.

95. Nervi B, Ramirez P, Rettig MP, Uy GL, Holt MS, Ritchey JK, Prior JL, Piwnica-worms D, Bridger G, Ley TJ, Dipersio JF. Chemosensitization of acute myeloid leukemia (AML) following mobilization by the CXCR4 antagonist AMD3100. Blood. 2009; 113:6206-15.
96. McCubrey JA, Steelman LS, Chappell WH, Abrams SL, Franklin RA, Montalto G, Cervello M, Libra M, Candido S, Malaponte G, Mazzarino MC, Fagone P, Nicoletti F, et al. Ras/Raf/MEK/ERK and PI3K/PTEN/Akt/mTOR cascade inhibitors: How mutations can result in therapy resistance and how to overcome resistance. Oncotarget. 2012; 3:1068111. https://doi.org/10.18632/oncotarget.659.

97. Mccubrey JA, Steelman LS, Chappell WH, Abrams SL, Wong EWT, Chang F, Lehmann B, Terrian DM, Milella M, Tafuri A, Stivala F, Libra M, Basecke J, et al. Roles of the Raf/MEK/ERK pathway in cell growth, malignant transformation and drug resistance. Biochim Biophys Acta. 2007; 1773:1263-84.

98. Sunters A, Madureira PA, Pomeranz KM, Sunters A, Madureira PA, Pomeranz KM, Aubert M, Brosens JJ, Cook SJ, Burgering BMT, Coombes RC, Lam EW. PaclitaxelInduced Nuclear Translocation of FOXO3a in Breast Cancer Cells Is Mediated by c-Jun NH 2 -Terminal Kinase and Akt Cancer Cells Is Mediated by c-Jun NH 2 -Terminal Kinase and Akt. Cancer Res. 2006; 66:212-20.

99. Lopez-Knowles E, Toole SAO, Mcneil CM, Millar EKA, Qiu MR, Crea P, Daly RJ, Musgrove EA, Sutherland RL. PI3K pathway activation in breast cancer is associated with the basal-like phenotype and cancer-specific mortality. Int $\mathbf{J}$ Cancer. 2010; 126:1121-31.

100. Epstein RJ. The CXCL12-CXCR4 chemotactic pathway as a target of adjuvant breast cancer therapies. Nat Rev Cancer. 2004; 4:1-9.

101. Cukierman E, Bassi DE. The mesenchymal tumor microenvironment. Cell Adh Migr. 2012; 6:285-96.

102. Wong D, Korz W. Translating an Antagonist of Chemokine Receptor CXCR4: From Bench to Bedside. Mol Pathways. 2008; 14:7975-81.

103. Saini KS, Loi S, de Azambuja E, Metzger-Filho O, Saini ML, Ignatiadis M, Dancey JE, Piccart-Gebhart MJ. Targeting the PI3K/AKT/mTOR and Raf/MEK/ERK pathways in the treatment of breast cancer. Cancer Treat Rev. 2013; 39:935-46.

104. Tavana H, Jovic A, Mosadegh B, Lee QY, Liu X, Luker KE, Luker GD, Weiss SJ, Takayama S. Nanoliter Liquid Patterning in Aqueous Environments for Spatially-Defined Reagent Delivery to Mammalian Cells. Nat Mater. 2009; 8:736-41.

105. Song JW, Cavnar SP, Walker AC, Luker KE, Gupta M, Tung YC, Luker GD, Takayama S. Microfluidic endothelium for studying the intravascular adhesion of metastatic breast cancer cells. PLoS One. 2009; 4.

106. Luker KE, Steele JM, Mihalko LA, Ray P, Luker GD. Constitutive and chemokine-dependent internalization and recycling of CXCR7 in breast cancer cells to degrade chemokine ligands. Oncogene. 2010; 29:4599-610.

107. Luker KE, Gupta M, Luker GD. Bioluminescent CXCL12 Fusion Protein for Cellular Studies of CXCR4 and CXCR7. Biotechniques. 2009; 47:625-32. 
108. Sato N, Maehara N, Goggins M. Gene Expression Profiling of Tumor - Stromal Interactions between Pancreatic Cancer Cells and Stromal Fibroblasts. Cancer Res. 2004; 64:6950-6.

109. Tejada ML, Yu L, Dong J, Jung K, Meng G, Peale FV, Frantz GD, Hall L, Liang X, Gerber H, Ferrara N. Tumordriven paracrine platelet-derived growth factor receptor $\alpha$ signaling is a key determinant of stromal cell recruitment in a model of human lung carcinoma. Clin Cancer Res. 2006; 12:2676-88.

110. Lall N, Henley-Smith CJ, De Canha MN, Oosthuizen CB, Berrington D. Viability Reagent, PrestoBlue, in Comparison with Other Available Reagents, Utilized in Cytotoxicity and Antimicrobial Assays. Int J Microbiol. 2013; 2013:1-5.
111. Yoav B, Hochberg Y. Controlling the False Discovery Rate: A Practical and Powerful Approach to Multiple Testing. J R Stat Soc. 1995; 57:289-300.

112. Matteson DS, James NA, Matteson DS, James NA, Approach AN. A Nonparametric Approach for Multiple Change Point Analysis of Multivariate Data. J Am Stat Assoc. 2014; 109:334-45.

113. Noble WS. How does multiple testing correction work? Nat Biotechnol. 2009; 27:1135-7.

114. Timmins NE, Nielsen LK. Generation of multicellular tumor spheroids by the hanging-drop method. Methods Mol Med. 2007; 140:141-51. 